\title{
Evidence for a nonplanar amplituhedron
}

\author{
Zvi Bern, ${ }^{a}$ Enrico Herrmann, ${ }^{b}$ Sean Litsey, ${ }^{a}$ James Stankowicz ${ }^{a}$ and Jaroslav $\operatorname{Trnka}^{b, c}$ \\ ${ }^{a}$ Department of Physics and Astronomy, UCLA, \\ Los Angeles, CA 90095, U.S.A. \\ ${ }^{b}$ Walter Burke Institute for Theoretical Physics, \\ California Institute of Technology, Pasadena, CA 91125, U.S.A. \\ ${ }^{c}$ Center for Quantum Mathematics and Physics (QMAP), \\ Department of Physics, University of California, Davis, CA 95616, U.S.A. \\ E-mail: bern@physics.ucla.edu, eherrmann@caltech.edu, \\ slitsey@ucla.edu, jjstankowicz@ucla.edu, trnka@ucdavis.edu
}

ABSTRACT: The scattering amplitudes of planar $\mathcal{N}=4$ super-Yang-Mills exhibit a number of remarkable analytic structures, including dual conformal symmetry and logarithmic singularities of integrands. The amplituhedron is a geometric construction of the integrand that incorporates these structures. This geometric construction further implies the amplitude is fully specified by constraining it to vanish on spurious residues. By writing the amplitude in a $d \log$ basis, we provide nontrivial evidence that these analytic properties and "zero conditions" carry over into the nonplanar sector. This suggests that the concept of the amplituhedron can be extended to the nonplanar sector of $\mathcal{N}=4$ super-Yang-Mills theory.

KEYwords: Scattering Amplitudes, Extended Supersymmetry

ARXiv EPrint: 1512.08591 


\section{Contents}

1 Introduction 1

2 Dual picture for planar integrands 4

2.1 Dual conformal symmetry 5

2.2 On-shell diagrams 8

$\begin{array}{ll}2.3 \text { Zero conditions from the amplituhedron } & 10\end{array}$

3 Nonplanar amplitudes $\quad 13$

3.1 Nonplanar conjectures 14

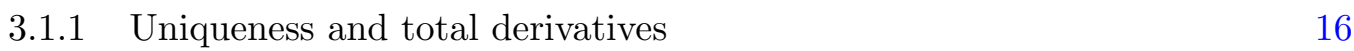

$\begin{array}{ll}3.2 & \text { Two-loop four-point amplitude } \\ 3.3 & \text { Three-loop four-point amplitude }\end{array}$

$\begin{array}{lll}3.3 & \text { Three-loop four-point amplitude } & 18\end{array}$

$\begin{array}{ll}\text { 3.3.1 Basis of unit leading singularity numerators } & 19\end{array}$

$\begin{array}{ll}3.3 .2 & \text { Matching the amplitude }\end{array}$

3.4 Two-loop five-point amplitude $\quad 23$

3.4.1 Basis of unit leading-singularity numerators 23

$\begin{array}{ll}3.4 .2 & \text { Matching the amplitude }\end{array}$

4 Zeros of the integrand $\quad 25$

$\begin{array}{lll}5 & \text { Conclusion } & 31\end{array}$

\section{Introduction}

Recent years have seen an enormous advance in our understanding of scattering amplitudes in $\mathcal{N}=4$ super-Yang-Mills (SYM) theory. Most progress has been made in the planar sector, with many calculations of new amplitudes with large numbers of loops and legs both at the integrand and integrated levels now available. Discoveries of new structures and symmetries have led to the development of deep theoretical frameworks which greatly aid new computations while also connecting to new areas of mathematics.

In the planar theory there are a number of recently discovered structures, including dual conformal symmetry [1-3], Yangian symmetry [4], integrability [5, 6], a dual interpretation in terms of Wilson loops [7-12], amplitudes at finite coupling using OPE [13-15], hexagon bootstrap [16-20], and symbols and cluster polylogarithmics [21-24], as well as a variety of other structures. More recently, scattering amplitudes were reformulated using on-shell diagrams and the positive Grassmannian [25-31] (see related work in ref. [32-35]). This reformulation fits nicely into the geometric concept of the amplituhedron [36] (see also refs. [37-43]), and makes connections to active areas of research in algebraic geometry and combinatorics (see e.g. refs. [44-49]). 
In this paper, we investigate how some of these properties carry over to the nonplanar sector. A basic difficulty in the nonplanar sector is that it is currently unclear how to define a unique integrand, largely due to the lack of global variables with which to describe a nonplanar integrand. Such ambiguities greatly obscure the desired structures that might be hiding in the amplitude. In addition, we lose Yangian symmetry and presumably any associated integrability constraints, as well as the connection between amplitudes and Wilson loops. Naively we also lose the ability to construct amplitudes using on-shell diagrams, the positive Grassmannian and the amplituhedron.

Nevertheless, one might suspect that many features of the planar theory can be extended to the full theory including nonplanar contributions. In particular, the conjectured duality between color and kinematics $[50,51]$ suggests that nonplanar integrands are obtainable directly from planar ones, and hence properties of the nonplanar theory should be related to properties of the planar sector. However, it is not a priori obvious which features can be carried over.

The dual formulation of planar $\mathcal{N}=4$ super-Yang-Mills scattering amplitudes using onshell diagrams and the positive Grassmannian makes manifest that the integrand has only logarithmic singularities, and can be written in a $d \log$ form. Furthermore, the integrand has no poles at infinity as a consequence of dual conformal symmetry. Recently, Arkani-Hamed, Bourjaily, Cachazo and one of the authors conjectured the same singularity properties hold to all loop orders for all maximally helicity violating (MHV) amplitudes in the nonplanar sector as well [52]. In a previous paper [53], we confirmed this explicitly for the full three-loop four-point integrand of $\mathcal{N}=4 \mathrm{SYM}$ by finding a basis of diagram integrands where each term manifests these properties. We also conjectured that to all loop orders the constraints give us the key analytic information contained in dual conformal symmetry. Additional evidence for this was provided from studies of the four- and five-loop amplitudes. These results then offer concrete evidence that analytic structures present in the planar amplitudes do indeed carry over to the nonplanar sector of the theory.

Now we take this further and show that in the planar case dual conformal invariance is equivalent to integrands with (i) no poles at infinity, and (ii) special values of leading singularities (maximal codimension residues). In the MHV sector, property (ii) and superconformal invariance imply that leading singularities are necessarily \pm 1 times the usual Parke-Taylor factor $[54,55]$. Moreover, the existence of a dual formulation using on-shell diagrams and the positive Grassmannian implies that (iii) integrands have only logarithmic singularities. While (i) and (iii) can be directly conjectured also for nonplanar amplitudes, property (ii) must be modified. As proven in ref. [56] for both planar and nonplanar cases, the leading singularities are linear combinations of Parke-Taylor factors with different orderings and with coefficients \pm 1 . This set of conditions was first conjectured in [52], and here we give a more detailed argument as to why the content of dual conformal symmetry in the MHV sector is exhausted by this set of conditions. We also provide direct nontrivial evidence showing they hold for the two-loop five-point amplitude and the three-loop fourpoint amplitude. While we might expect this structure to hold in the MHV sector, beyond this we expect $R$ invariants [3] to play an important role.

The main purpose of this paper is to present evidence for the amplituhedron concept [36] beyond the planar limit. The amplituhedron is defined in momentum twistor 
variables which intrinsically require cyclic ordering of amplitudes, making direct nonplanar tests in these variables impossible. However, we can test specific implications even for nonplanar amplitudes. In ref. [40], Arkani-Hamed, Hodges and one of the authors argued that the existence of the "dual" amplituhedron implies certain positivity conditions of amplitude integrands. Indeed, these conditions were proven analytically for some simple cases and numerically in a large number of examples. (Interestingly, these conditions appear to hold even post-integration $[40,57])$. The dual amplituhedron can be interpreted as a geometric region of which the amplitude is literally a volume, in contrast to the original definition where the amplitude is a form with logarithmic singularities on the boundaries of the amplituhedron space. This implies a very interesting property when the integrand is combined into a single rational function: its numerator represents a codimension one surface which lies outside the dual amplituhedron space. The surface is simply described as a polynomial in momentum twistor variables and therefore can be fully determined by the zeros of the polynomial, which correspond to points violating positivity conditions defining the amplituhedron. A nontrivial statement implied by the amplituhedron geometry is that all these zeros can be interpreted as cuts where the amplitude vanishes.

This leads to a concrete feature that can be tested even in a diagrammatic representation of a nonplanar amplitude:

\section{The integrand should be determined entirely from homogeneous conditions, up to an overall normalization.}

Concretely, by "homogeneous conditions" we mean the conditions of no poles at infinity, only logarithmic singularities, and also unitarity cuts that vanish. That is, in the unitarity method, the only required cut equations are the ones where one side of the equation is zero, as opposed to a nontrivial kinematical function. These zeros occur either because the amplitude vanishes on a particular branch of the cut solutions or because the cut is spurious. ${ }^{1}$ This conjecture has exciting implications because this feature is closely related to the underlying geometry in the planar sector, suggesting that the nonplanar contributions to amplitudes admit a similar structure.

To test this conjecture we use the three-loop four-point and two-loop five-point nonplanar amplitudes as nontrivial examples. A key assumption is that the desired properties can all be made manifest diagram-by-diagram [53]. While it is unknown if this assumption holds for all amplitudes at all loop orders, at the relatively low loop orders that we work our results confirm that this is a good hypothesis. The three-loop four-point integrand was first obtained in ref. [58], while the two-loop five-point integrand was first calculated in ref. [59] in a format that makes the duality between color and kinematics manifest. Here we construct different representations that make manifest that the amplitudes have only logarithmic singularities and no poles at infinity. These representations are then compatible with the notion that there exists a nonplanar analog of dual conformal symmetry and a geometric formulation of nonplanar amplitudes. We organize the amplitudes in terms of basis integrands that have only \pm 1 leading singularities. The coefficient of these integrals

\footnotetext{
${ }^{1} \mathrm{~A}$ spurious cut is one that exposes a non-physical singularity, i.e. a singularity that is not present in the full amplitude.
} 
in the amplitudes are then simply sums of Parke-Taylor factors, as proved in ref. [56]. We also show that homogeneous conditions are sufficient to determine both amplitudes up to an overall factor, as expected if a nonplanar analog of the amplituhedron were to exist.

This paper is organized as follows. In section 2 we summarize properties connected to the amplituhedron picture of amplitudes in planar $\mathcal{N}=4 \mathrm{SYM}$. Then in section 3 we turn to a discussion of properties of nonplanar amplitudes, showing in various examples that the consequences of dual conformal invariance and the logarithmic singularity condition do carry over to the nonplanar sector. Finally, in section 4 we give evidence for a geometric interpretation of the amplitude by showing that the coefficients in the diagrammatic expansion are determined by zero conditions. In section 5 we give our conclusions.

\section{Dual picture for planar integrands}

In this section we summarize known properties of planar amplitudes in $\mathcal{N}=4 \mathrm{SYM}$ theory that we wish to carry beyond the planar limit to amplitudes of the full theory. We emphasize those features associated with the amplituhedron construction. In the planar case, we strip the amplitude of color factors. Later when we deal with the nonplanar case, we restore them.

The classic representation of scattering amplitudes uses Feynman diagrams. At loop level the diagrams can be expressed in terms of scalar and tensor integrals. We can then write the amplitude as ${ }^{2}$

$$
\mathcal{M}=\sum_{j} d_{j} \int d \mathcal{I}^{j}
$$

where the sum is over a set of basis integrands $d \mathcal{I}^{j}$ and $d_{j}$ are functions of the momenta of external particles, hereafter called kinematical functions. In general the integrations should be performed in $D=4-2 \epsilon$ dimensions as a means for regulating both infrared and ultraviolet divergences. While the integrand can contain pieces that differ between four dimensions and $D$ dimensions, in the present paper we ignore any potential contributions proportional to $(-2 \epsilon)$ components of loop momenta. At four-points we do not expect any such contribution through at least six loops [60], but they can enter at lower loop orders as the number of legs increases [61]. We will not deal with such contributions in this paper, but we expect that they can be treated systematically as corrections to any uncovered four-dimensional structure.

In $\mathcal{N}=4$ SYM we can split off an MHV prefactor, including the supermomentum conserving delta function $\delta^{8}(Q)$, from all $d_{j}$,

$$
\operatorname{PT}(1234 \cdots n)=\frac{\delta^{8}(Q)}{\langle 12\rangle\langle 23\rangle\langle 34\rangle \cdots\langle n 1\rangle},
$$

which defines a Parke-Taylor factor $[54,55]$. Usually, we describe the $d \mathcal{I}^{j}$ in terms of local integrals that share the same Feynman propagators as corresponding Feynman diagrams.

\footnotetext{
${ }^{2}$ In general we drop overall factors of $1 /(2 \pi)^{D}$ and couplings from the amplitude, since these play no role in our discussion.
} 
However, in the planar sector of the amplitude we do not need to rely on those diagrams. Instead we can choose dual coordinates $k_{i}=x_{i}-x_{i-1}$ to encode external kinematics, as well as analogously defined $y_{j}$ for different loop momenta. The variables are associated with the faces of each diagram, are globally defined for all diagrams, and allow us to define a unique integrand by appropriately symmetrizing over the faces [31]. With these variables, we can sum all diagrams under one integration symbol and write an $L$-loop amplitude as

$$
\mathcal{M} \sim \int d \mathcal{I}\left(x_{i}, y_{j}\right)=\int d^{4} y_{1} d^{4} y_{2} \ldots d^{4} y_{L} \mathcal{I}\left(x_{i}, y_{j}\right),
$$

where $d \mathcal{I}$ is the integrand form and $\mathcal{I}$ is the unique integrand of the scattering amplitude. The integrand form $d \mathcal{I}$ for the $n$-point amplitude is a unique rational function with many extraordinary properties that we will review in this section. Particularly effective ways of constructing the integrand are unitarity cut methods [62-64] or BCFW recursion relations $[31,65]$.

\subsection{Dual conformal symmetry}

A key property of $\mathcal{N}=4 \mathrm{SYM}$ planar amplitudes is that they possess dual conformal symmetry [1-3]. This symmetry acts like ordinary conformal symmetry on the dual variables $x_{i}$ and $y_{j}$ mentioned above. This can be supersymmetrically extended to a dual superconformal symmetry, and in combination with the ordinary superconformal symmetry it closes into the infinite dimensional Yangian symmetry [4]. This is a symmetry of tree-level amplitudes, and at loop level is a symmetry of quantities such as the integrand $d \mathcal{I}$, and IR safe quantities like ratio functions [3].

We are interested in understanding the implications of dual conformal symmetry on the analytic structure of the amplitude. Good variables for doing so are the momentum twistor variables $Z_{i}$, introduced in ref. [66]. These are points in complex projective space $\mathbb{C P}^{3}$ and are related to the spinor helicity variables $\left.\lambda_{i} \equiv|i\rangle, \widetilde{\lambda}_{i} \equiv \mid i\right]$ via

$$
Z_{i}=\left(\begin{array}{c}
\lambda_{i} \\
\mu_{i}
\end{array}\right) \quad \text { where } \mu_{i}^{\dot{a}}=x_{i}^{a \dot{a}} \lambda_{i, a},
$$

where $x_{i}^{a \dot{a}}$ are the dual variables defined above in spinor indices. The set of $n$ on-shell external momenta are then described by $n$ momentum twistors $Z_{i}, i=1,2, \ldots, n$. Momentum twistors are unconstrained variables and trivialize momentum conservation, which is a quadratic condition on the $\lambda_{i}, \widetilde{\lambda}_{i}$ spinors. Each off-shell loop momentum $\ell_{i}$ is equivalent to a point $y_{i}$ in dual momentum space, which in turn is represented by a line $Z_{A_{i}} Z_{B_{i}}$ in momentum twistor space.

Dual conformal symmetry acts as $\mathrm{SL}(4)$ on $Z_{i}$, and we can construct invariants from a contraction of four different $Z$ 's,

$$
\langle i j k l\rangle \equiv\left\langle Z_{i} Z_{j} Z_{k} Z_{l}\right\rangle=\epsilon_{\alpha \beta \rho \sigma} Z_{i}^{\alpha} Z_{j}^{\beta} Z_{k}^{\rho} Z_{l}^{\sigma} .
$$

Any dual conformal invariant can be written using these four-brackets. The contractions of spinor helicity variables $\lambda$ can be written as

$$
\langle i j\rangle \equiv \epsilon_{a b} \lambda_{i}^{a} \lambda_{j}^{b}=\epsilon_{\alpha \beta \rho \sigma} Z_{i}^{\alpha} Z_{j}^{\beta} I^{\rho \sigma},
$$


where $I^{\rho \sigma}$ is the infinity twistor defined in ref. [66]. An expression containing $I^{\rho \sigma}$ breaks dual conformal symmetry because $I^{\rho \sigma}$ does not transform as a tensor. There is a simple dictionary between momentum space and momentum twistor invariants; we refer the reader to ref. [66] for details.

A simple example of a dual conformal invariant integrand is the zero-mass box,

$$
d \mathcal{I}=\frac{d^{4} \ell\left(k_{1}+k_{2}\right)^{2}\left(k_{2}+k_{3}\right)^{2}}{\ell^{2}\left(\ell-k_{1}\right)^{2}\left(\ell-k_{1}-k_{2}\right)^{2}\left(\ell+k_{4}\right)^{2}}=\frac{\left\langle A B d^{2} A\right\rangle\left\langle A B d^{2} B\right\rangle\langle 1234\rangle^{2}}{\langle A B 12\rangle\langle A B 23\rangle\langle A B 34\rangle\langle A B 41\rangle} .
$$

This represents the full one-loop four-point integrand form in $\mathcal{N}=4 \mathrm{SYM}$. Note that the integrand in eq. (2.7) is completely projective in all variables $Z$, and the infinity twistor is absent in this expression. This is true for any dual conformal invariant integrand.

This brings us to a key question we would like to answer here:

What is the content of dual conformal symmetry for momentum-space integrands?

In momentum twistor space the answer is obvious: the infinity twistor $I^{\rho \sigma}$ is absent. Suppose instead the infinity twistor is present. What is the implication in momentum space? The first trivial case is when the prefactor of the integrand is not chosen properly. For example, if the factor $\left(k_{2}+k_{3}\right)^{2}$ in the numerator of the zero-mass box in eq. (2.7) is replaced with say $\left(k_{1}+k_{2}\right)^{2}$, this will introduce a dependence on $I$, signaling broken dual conformal invariance. In this case, the only dependence on the infinity twistor is through four-brackets $\langle i j I\rangle$ involving only external variables. The presence of these is easily avoided by correctly normalizing $d \mathcal{I}$.

The nontrivial interesting cases occur when the infinity twistor appears in combination with the line $Z_{A} Z_{B}$ that represents a loop momentum, e.g. $\langle A B I\rangle$. In this case no prefactor depending only on external kinematics can fix it, and the integrand form necessarily violates dual conformal symmetry. The factor $\langle A B I\rangle$ (or its powers) can appear either in the numerator or the denominator. If it is in the denominator, the integrand has a spurious singularity at $\langle A B I\rangle=0$. In momentum space this corresponds to sending $\ell \rightarrow \infty$. To see this, consider a simple example: the one-loop triangle given by

$$
d \mathcal{I}=\frac{d^{4} \ell\left(k_{1}+k_{2}\right)^{2}}{\ell^{2}\left(\ell-k_{1}\right)^{2}\left(\ell-k_{1}-k_{2}\right)^{2}}=\frac{\left\langle A B d^{2} A\right\rangle\left\langle A B d^{2} B\right\rangle\langle 1234\rangle\langle 23 I\rangle}{\langle A B 12\rangle\langle A B 23\rangle\langle A B 34\rangle\langle A B I\rangle} .
$$

If we parametrize the loop momentum as $\ell=\alpha \lambda_{1} \widetilde{\lambda}_{1}+\beta \lambda_{2} \widetilde{\lambda}_{2}+\gamma \lambda_{1} \widetilde{\lambda}_{2}+\delta \lambda_{2} \widetilde{\lambda}_{1}$ and send $\gamma \rightarrow \infty$ while keeping $\gamma \delta=$ finite, there is a pole which corresponds to $\ell \rightarrow \infty$. Bubble integrals even have a double pole at infinity, which corresponds to a double pole $\langle A B I\rangle^{2}$ when written in momentum twistor space.

If the $\langle A B I\rangle$ factor is in the numerator there is a problem with the values of leading singularities. For an $L$-loop integrand these are $4 L$-dimensional residues that are just rational functions of external kinematics [67]. If the integrand form is dual conformal invariant, all its leading singularities are dual conformal cross ratios (defined in ref. [68]). A special case is when they are all \pm 1 , as for the box integrand in eq. (2.7). 
If the integrand has $\langle A B I\rangle$ in the numerator, the values of leading singularities, denoted $L S(\cdot)$, depend on $\left\langle(A B)^{*} I\right\rangle$,

$$
L S(d \mathcal{I})=\left\langle(A B)^{*} I\right\rangle \cdot \mathcal{F}\left(Z_{i},\langle a b\rangle\right)
$$

where $(A B)^{*}$ is the position of the line $A B$ with the leading singularity solution substituted in. The function $\mathcal{F}$ is dual conformal invariant up to some two-brackets of external twistors $\langle a b\rangle$ from normalization. For one particular leading singularity we can choose the normalization of $d \mathcal{I}$ and therefore force $\mathcal{F}$ to cancel $\left\langle(A B)^{*} I\right\rangle$, restoring dual conformal symmetry. However, different leading singularities - of which each integrand has at least two by the residue theorem - are located at different $(A B)^{*}$ so that it is not possible to simultaneously normalize all leading singularities correctly using only external data. As a result, some of the leading singularities necessarily are not dual conformal invariant. A simple example is the scalar one-loop pentagon,

$$
\begin{aligned}
d \mathcal{I} & =\frac{d^{4} \ell\left(k_{1}+k_{2}\right)^{2}\left(k_{2}+k_{3}\right)^{2}\left(k_{3}+k_{4}\right)^{2}}{\ell^{2}\left(\ell-k_{1}\right)^{2}\left(\ell-k_{1}-k_{2}\right)^{2}\left(\ell-k_{1}-k_{2}-k_{3}\right)^{2}\left(\ell+k_{5}\right)^{2}} \\
& =\frac{\left\langle A B d^{2} A\right\rangle\left\langle A B d^{2} B\right\rangle\langle A B I\rangle\langle 1234\rangle\langle 2345\rangle\langle 5123\rangle}{\langle A B 12\rangle\langle A B 23\rangle\langle A B 34\rangle\langle A B 45\rangle\langle A B 51\rangle\langle 23 I\rangle},
\end{aligned}
$$

which is not dual conformal invariant, as implied by the appearance of the infinity twistor. The numerator of this pentagon can be modified to a chiral version studied in ref. [69], which restores dual conformal symmetry.

Based on these considerations, we can summarize the content of dual conformal symmetry of individual integrands in momentum space in two conditions:

1. There are no poles as $\ell \rightarrow \infty$.

2. All leading singularities are dual conformal cross ratios.

Any integrand that satisfies these properties necessarily is dual conformal invariant.

In the context of integrands for MHV amplitudes in planar $\mathcal{N}=4 \mathrm{SYM}$, if we strip off the MHV tree-level amplitude, i.e. the Parke-Taylor factor PT(123 ...n) eq. (2.2),

$$
\mathcal{M}=\operatorname{PT}(123 \ldots n) \int d \mathcal{I}
$$

then the integrand $d \mathcal{I}$ is dual conformal invariant satisfying both properties above. There are even stronger constraints: superconformal symmetry requires that all leading singularities are holomorphic functions [70] of $\lambda_{i}$ 's alone. The only functions that are holomorphic, satisfy property 2 above, and have the correct mass dimension and little-group weight are pure numbers. In the normalization conventions adopted here, they are \pm 1 or 0 . While we do not have a direct formulation of dual conformal symmetry in the nonplanar sector, we shall find analogous analytic structures in the amplitudes for all the examples we study. The role of the Parke-Taylor factor will have to be modified slightly however. 

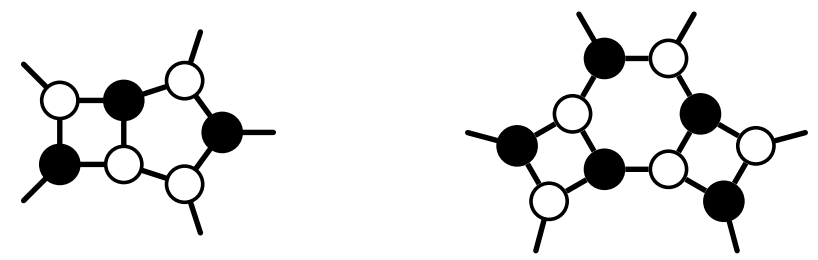

Figure 1. Sample on-shell diagrams. The black and white dots respectively represent MHV and $\overline{\mathrm{MHV}}$ three-point amplitudes. Black lines are on-shell particles.

\subsection{On-shell diagrams}

On-shell diagrams provide another novel representation of the integrand [25]. These are diagrams with black and white vertices connected by lines, as illustrated in figure 1. Black vertices represent MHV three-point amplitudes, white vertices $\overline{M H V}$ three-point amplitudes, and all lines, both internal and external, represent on-shell particles. There are two indices associated with any on-shell diagram: the number of external legs $n$ and the helicity index $k$. The $k$-index is defined as

$$
k=\sum_{V} k_{V}-P,
$$

where the sum is over all vertices $V, k_{V}$ is the $k$-count of the tree-level amplitude in a given vertex, and $P$ is the number of on-shell internal propagators. Black and white vertices have $k_{B}=2$ and $k_{W}=1$, respectively. As an example, the first diagram in figure 1 has $k=(2+2+2)+(1+1+1+1)-8=2$. This $k$ corresponds to the total number of external negative helicities.

The values of the diagrams are computed by integrating over the phase space $d \Omega_{i}$ of on-shell internal particles the product of tree-level amplitudes $A_{j}$ for each vertex

$$
d \Omega=\prod_{i} \int d \Omega_{i} \prod_{j} A_{j}
$$

An on-shell diagram may be interpreted as a specific generalized unitarity cut of an amplitude. In this interpretation, the internal lines of an on-shell diagram represent cut propagators. The on-shell diagram represents a nonvanishing valid cut of the amplitude only if the labels $n, k$ of the on-shell diagram coincide with the same labels of the amplitude.

A very different way to describe and calculate planar on-shell diagrams is as cells of a positive Grassmannian $G_{+}(k, n)$ [25]. For each diagram we define variables $\alpha_{j}$ associated with edges or faces of the diagram. Using certain rules [25], we build a $(k \times n)$ matrix $C$ with positive main minors - a cell in the positive Grassmannian. Then the value of the diagram is given by a logarithmic form in the variables of the diagram, multiplied by a delta function which connects the $C$ matrix with external variables (ordinary momenta or momentum twistors), ${ }^{3}$

$$
d \Omega=\frac{d \alpha_{1}}{\alpha_{1}} \frac{d \alpha_{2}}{\alpha_{2}} \frac{d \alpha_{3}}{\alpha_{3}} \ldots \frac{d \alpha_{m}}{\alpha_{m}} \delta(C \cdot Z) .
$$

\footnotetext{
${ }^{3}$ We suppress wedge notation for forms throughout: $d x d y \equiv d x \wedge d y$.
} 
This is known as a " $d \log$ form" since all singularities have the structure $d \log \alpha_{i} \equiv d \alpha_{i} / \alpha_{i}$. For further details we refer the reader to ref. [25].

Since the planar integrand can be expressed as a sum of these on-shell diagrams via recursion relations [25], all its singularities are also logarithmic. That is, if we approach a singularity of the amplitude for $\alpha_{j} \rightarrow 0$ the integrand develops a pole

$$
d \mathcal{I} \stackrel{\alpha_{j}=0}{\longrightarrow} \frac{d \alpha_{j}}{\alpha_{j}} d \widetilde{\mathcal{I}} \quad \text { where } d \widetilde{\mathcal{I}} \text { does not depend on } \alpha_{j}
$$

This property is not at all obvious in more traditional diagrammatic representations of scattering amplitudes.

The on-shell diagrams are individually both dual conformal and Yangian invariant and therefore are good building blocks that make both symmetries manifest. On the other hand, rewriting the variables $\alpha_{j}$ in terms of momenta results in spurious poles which only cancel in the sum over all contributions.

While eq. (2.15) holds for all planar $\mathcal{N}=4$ integrands for all helicities, in general the variables $\alpha_{j}$ are variables of on-shell diagrams that are nontrivially related to the loop and external variables through the delta function $\delta(C \cdot Z)$. For MHV, NMHV (next-to-MHV) and $\mathrm{N}^{2} \mathrm{MHV}$ (next-to-next-to-MHV), this change of variables implies that the integrand also has logarithmic singularities directly in momentum space. For higher $\mathrm{N}^{m} \mathrm{MHV}$ amplitudes with $m>2$, the fermionic Grassmann variables enter in the change of variables so that the integrand is not a $d \log$ form in momentum variables directly. In this paper, we only deal with the case of MHV amplitudes, so that the $d$ log structure is straightforwardly visible in momentum space. As conjectured in ref. [52], the same properties hold at the nonplanar level.

Pure integrand diagrams. In the MHV sector, we can check the $d$ log property for individual momentum-space planar diagrams with only Feynman propagators. In this check, we consider different cuts $^{4}$ of a diagram and probe whether eq. (2.15) is always valid in momentum space. If so, its integrand form indeed has logarithmic singularities and can in principle be written as a sum of $d \log$ forms

$$
d \mathcal{I}^{j}=\sum_{k} b_{k} d \log f_{1}^{(k)} d \log f_{2}^{(k)} \ldots d \log f_{4 L}^{(k)}
$$

where $f_{m}^{(k)}$ are some functions of external and loop momenta. Constraining these integrands to be dual conformal invariant further enforces that the functions $d \log f_{m}^{(k)}$ never generate a pole if any of the loop momenta approach infinity, $\ell_{i} \rightarrow \infty$. In addition, for appropriately normalized diagrams the coefficients $b_{k}$ are all equal to \pm 1 . A form $d \mathcal{I}^{j}$ with all these properties is called a pure integrand form. A simple example of such a form is the box integrand in eq. (2.7) which can be expressed explicitly as a single $d$ log form [25]. More complicated $d \log$ integrands have been used to write explicit expressions for one-loop and two-loop planar integrands for all multiplicities [71, 72]. Whenever the amplitude is built

\footnotetext{
${ }^{4}$ We use the words "cuts" and "residues" interchangeably throughout this paper.
} 
from $d \mathcal{I}^{j}$ 's that are individually pure integrands, we will refer to such an expansion as a pure integrand representation of the amplitude, and to the set of $d \mathcal{I}^{j}$ 's as a pure integrand basis.

We can now expand the $n$-point planar MHV integrand with Parke-Taylor tree amplitudes factored out as a sum of pure integrands,

$$
d \mathcal{I}=\sum_{j} a_{j} d \mathcal{I}^{j}
$$

The existence of a diagram basis of pure integrands $d \mathcal{I}^{j}$ with only local poles is a conjecture. There is no guarantee that we can fix the $a_{j}$ coefficients of this ansatz to match the integrand of the amplitude; it might have been necessary to use non-pure integrands where unwanted singularities cancel between diagrams. Presently, it seems that pure integrands are sufficient up to relatively high loop order. The coefficients must all be $a_{j}= \pm 1,0$ based on the requirements of superconformal and dual conformal symmetry. Their precise values are determined by calculating leading singularities or other unitarity cuts.

We note that the representation in eq. (2.17) does not make the full Yangian symmetry manifest, as there is a tension between this symmetry and locality. However, the representation does make manifest both dual conformal symmetry and logarithmic singularities.

\subsection{Zero conditions from the amplituhedron}

With on-shell diagrams, scattering amplitudes are built from abstract mathematical objects with no reference to spacetime dynamics. This is an important step towards finding a new description of physics where locality and unitarity are not fundamental, but rather are derived from geometric properties of amplitudes. The on-shell diagrams individually have this flavor, but the particular sum that gives the amplitude is dictated by recursion relations that are based on unitarity properties. A procedure that dictates which particular sum of on-shell diagrams gives the amplitude without reference to unitarity would therefore be an improvement on recursion relations. The amplituhedron exactly has this property [36] as it is a self-consistent geometric definition of the planar integrand. Here we will not need the details of this object, just some of its basic properties.

We focus mainly on the fact that the integrand of scattering amplitudes is defined as a differential form $d \Omega$ with logarithmic singularities on the boundaries of the amplituhedron space. This space is defined as a certain map of the positive Grassmannian through the matrix of positive (bosonized) external data $Z$ for the tree-level case, and its generalization to loops. A particular representation of the amplitude in terms of on-shell diagrams provides a triangulation of this space, but the definition of the amplituhedron is independent of any particular triangulation.

The underlying assumptions in this construction are logarithmic singularities, in terms of which the form $d \Omega$ is defined, and dual conformal symmetry, which is manifest in momentum twistor space and generalizations thereof. All other properties of the integrand, including locality and unitarity, are derived from the amplituhedron geometry. This gives a complete definition of the integrand in a geometric language; yet, as mentioned in ref. [40], it is desirable to find another formulation which calculates the integrand as a volume of an object rather than as a differential form with special properties. In search of this dual 
amplituhedron it was conjectured in ref. [40] and checked in a variety of cases that the integrand $\mathcal{I}$ (without the measure) is positive when evaluated inside the amplituhedron. This is exactly the property we expect to be true for a volume function. If we write $\mathcal{I}$ as a numerator divided by all local poles,

$$
\mathcal{I}=\frac{N}{\left.\prod \text { (local poles }\right)},
$$

then, since $N$ is a polynomial in the loop variables $(A B)_{j}$ (and for non-MHV cases also in other objects), it must be completely fixed by its zeros (roots). An interesting conjecture is that the zeros of $N$ have two simple interpretations:

- The zeros correspond to forbidden cuts generated by the denominator; geometrically these are points outside the amplituhedron.

- The zeros cancel higher poles in the denominator to ensure that all singularities are logarithmic.

This should be true for all singularities of the integrand, both in external and loop variables. In the context of MHV amplitudes however, only the loop part is nontrivial. As an example, we can write the MHV one-loop integrand in the following way,

$$
\mathcal{I}=\frac{N\left(A B, Z_{i}\right)}{\langle A B 12\rangle\langle A B 23\rangle\langle A B 34\rangle \ldots\langle A B n 1\rangle},
$$

where $N\left(A B, Z_{i}\right)$ is a degree $n-4$ polynomial in $A B$ with proper little group weights in $Z_{i}$. In this case the denominator generates only logarithmic poles on the cuts, and the numerator $N$ is completely fixed (up to an overall constant) only by requiring that it vanishes on all forbidden cuts. There are two types of forbidden cut solutions for MHV amplitudes:

- Unphysical cut solutions: all helicity amplitudes vanish. In the on-shell diagram representation: no on-shell diagram exists.

- Non-MHV cut solutions: only MHV amplitudes vanish while other helicity amplitudes can be non-zero. In the on-shell diagram representation: the corresponding on-shell diagram has $k \neq 2$.

A simple example of the first case is the collinear cut $\ell=\alpha k_{1}$ followed by cutting another propagator $\left(\ell-k_{1}-k_{2}-k_{3}\right)^{2}$ of the pentagon integral in eq. (2.10). In momentum twistor geometry this corresponds to $\langle A B 12\rangle=\langle A B 23\rangle=\langle A B 45\rangle=0$ (as well as setting a Jacobian to zero) which localizes $Z_{A}=Z_{2}, Z_{B}=(123) \cap(45)$. This is an example of an unphysical cut which vanishes for all amplitudes including MHV, and the numerator $N$ in eq. (2.19) vanishes for this choice of $Z_{A}, Z_{B}$.

All forbidden cuts correspond to points outside the amplituhedron and therefore we can think about $N$ as a codimension one surface outside the amplituhedron. The amplituhedron and the surface $N$ can only touch on lower dimensional boundaries. This is 


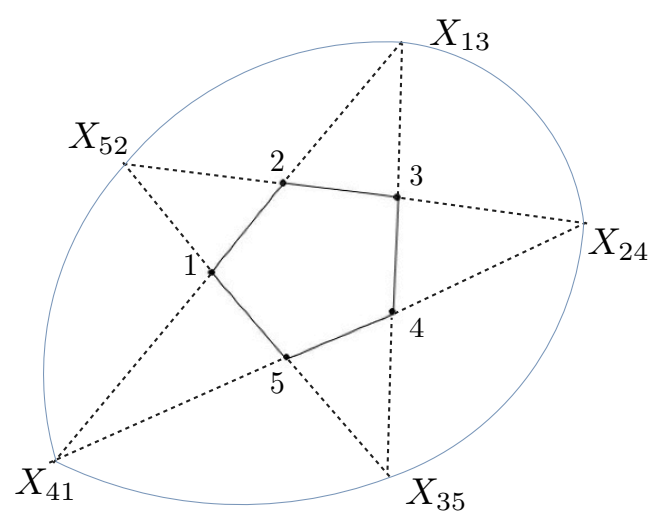

Figure 2. A simple amplituhedron example [40]. The area of the pentagon formed by the black solid line is the amplituhedron. The points $X_{i, i+2}$ define zeros of a numerator, so the conic given by the outer (blue) solid lines connecting the points represents the numerator.

completely consistent with the picture of the amplitude being the actual volume of the dual amplituhedron, making a clear distinction between inside and outside of the space.

Consider the simple example discussed in ref. [40] and shown in figure 2. In this case the amplituhedron is the area of the pentagon. The numerator $N$ is given by the conic that passes through five given points cyclically labeled by the $X_{i, i+2}$. These points correspond to "unphysical" singularities of the form $d \Omega$. Knowing the positions of the $X_{i, i+2}$ fully fixes the numerator $N$ as there is a unique conic passing through five points. Knowing $N$ fixes the integrand $\mathcal{I}$, per eq. (2.18). Note that all five $X_{i, i+2}$ are outside the amplituhedron (in this case the pentagon). The existence of a zero surface outside the amplituhedron in this example directly leads to a geometric construction of the integrand. The same happens for more complicated amplituhedra, which may lack such an intuitive visualization.

Now let us go several steps back and consider the standard expansion for the integrand, eq. (2.17), in momentum space as the starting point, and think about the zero conditions as coming from physics (unphysical cuts) rather than geometry (forbidden boundaries). We can reformulate the conjecture about fixing $N$ in eq. (2.18) in terms of unknown coefficients $a_{j}$ in the expansion in eq. (2.17):

\section{All coefficients $a_{j}$ are fixed by zero conditions, up to an overall normalization.}

By zero conditions we mean both unphysical and non-MHV cuts (as defined above) for which the integrand vanishes, $0=\left.d \mathcal{I}\right|_{\text {cuts }}$. The overall normalization just means the overall scale of the amplitude is one undetermined coefficient of the $a_{j}$, which may be fixed by one non-zero condition.

Assuming the integrand may be expanded as in eq. (2.17) automatically assumes the presence of only logarithmic singularities as well as the cancellation of some unphysical cuts, viz. those which do not correspond to planar diagrams. On one hand, we can think about this conjecture as a reduced version of the one stated in ref. [40] where both logarithmic singularities and diagram-like cuts were nontrivial conditions on the numerator $N$ of the planar integrand eq. (2.18). On the other hand, a (dual) amplituhedron exactly implies 


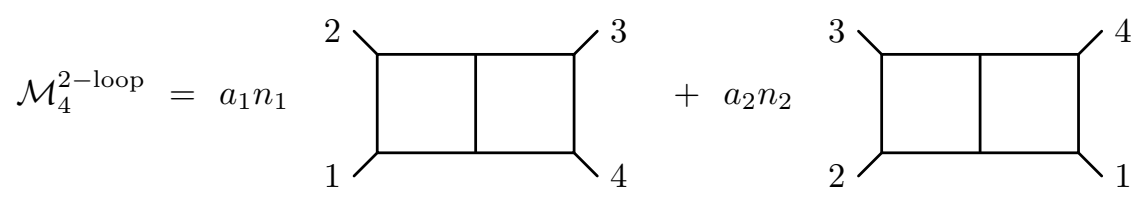

Figure 3. The planar two-loop four-point amplitude can be represented in terms of double-box diagrams.

our conjecture about zero conditions given the diagrammatic expansion of the integrand in eq. (2.17). And most importantly, our new conjecture is now formulated in a language which allows us to carry it over to the nonplanar sector later in the paper.

A first simple example that illustrates our zero conditions conjecture is the planar twoloop four-point amplitude [73], which can be represented using the diagrammatic expansion in figure 3. The diagrams represent the denominators of individual integrands and their unit leading singularity normalizations are $n_{1}=s^{2} t, n_{2}=s t^{2}$. The overall planar ParkeTaylor factor PT(1234) is suppressed. We can consider a simple non-MHV cut on which the amplitude should vanish and relate the coefficients as $a_{1}=a_{2}$, which is indeed correct. We will elaborate on this example in section 4 in the context of nonplanar amplitudes where more diagrams contribute.

\section{Nonplanar amplitudes}

As already noted, there is an essential difference between the planar and nonplanar sectors. In the nonplanar case, it is not known how to construct a unique integrand prior to integration. This is a direct consequence of the lack of global variables. Without those, the choice of variables in one nonplanar diagram relative to the choice in another diagram is arbitrary. This is a nontrivial obstruction to carrying over the planar amplituhedron construction directly to the full amplitude.

Here we circumvent this problem and follow the same strategy as in refs. [52, 53], which is to consider diagrams as individual objects and to impose all desired properties diagram-by-diagram. These elements then form a basis for the complete amplitude and give us a representation in terms of a linear combination of said objects. Each integral is furthermore dressed by color factors $c_{j}$ and with some kinematical coefficients $d_{j}$ that need to be determined,

$$
\mathcal{M}=\sum_{j} d_{j} c_{j} \int d \mathcal{I}^{j}
$$

The individual pieces $d \mathcal{I}^{j}$ interpreted as integrand forms are not really well defined because of the arbitrariness in their choice of variables, and they become well-defined only when integrated over loop momenta. However, we can still impose nontrivial requirements on the singularity structure of individual diagrams as was done in refs. [52, 53]. This is because unitarity cuts of the amplitude impose constraints in terms of a well defined set of cut momenta, just as they do in the planar sector. This implies that the integrand forms 


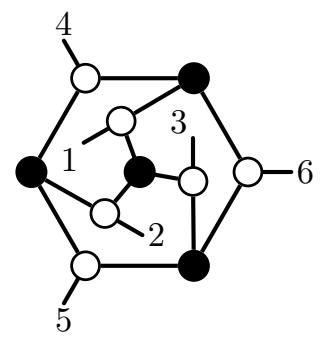

Figure 4. Example of a nonplanar on-shell diagram.

$d \mathcal{I}^{j}$ are interesting in their own right and that we can systematically study their properties with the tools at hand. In particular, we will see concrete examples where MHV integrands may be expanded in a pure integrand basis.

\subsection{Nonplanar conjectures}

In the context of $\mathcal{N}=4 \mathrm{SYM}$ it is natural to propose the following properties of the "integrand" even in nonplanar cases:

(i) The integrand has only logarithmic singularities.

(ii) The integrand has no poles at infinity.

(iii) The leading singularities of the integrand all take on special values.

The presence of only logarithmic singularities (i) would be an indication of the "volume" interpretation of nonplanar amplitudes. We will give more detailed evidence for such an interpretation in the next section. Demonstrating properties (ii) and (iii) would provide nontrivial evidence for the existence of an analog of dual conformal symmetry for full $\mathcal{N}=4 \mathrm{SYM}$ amplitudes, including the nonplanar sector. Since we lack nonplanar momentum twistor variables we cannot formulate an analogous symmetry directly, yet the basic constraints of properties (ii) and (iii) on nonplanar amplitudes would be identical to the constraints of dual conformal symmetry on planar amplitudes.

The first property (i) can be directly linked to the properties of on-shell diagrams, which are well-defined beyond the planar sector $[38,56]$. Nonplanar on-shell diagrams, one of which is illustrated in figure 4, are calculated following the same rules as in the planar case [25]. In particular, they are given by the same logarithmic form eq. (2.14), where the $C$-matrix is now some cell in the (not necessarily positive) Grassmannian $G(k, n)$. However, the singularities are again logarithmic and for MHV, NMHV, and $\mathrm{N}^{2} \mathrm{MHV}$ amplitudes; this property holds directly in momentum space like in the planar case. At present it is not known whether this is a property of the full amplitude, including nonplanar contributions. Unlike the planar case, we do not currently have an on-shell diagram representation of the amplitude since it is not known how to unambiguously implement recursion relations. If such a construction exists then the amplitude would share the properties of the on-shell diagrams, including their singularity structure. Therefore it is very natural to conjecture that the full amplitude indeed has only logarithmic singularities [52]. 
Because there is no global definition for the integrand, it is reasonable to assume that there exist $d \mathcal{I}^{j}$ as in eq. (3.1) such that each $d \mathcal{I}^{j}$ has only logarithmic singularities [53]. That is, we assume that there exists a $d \log$ representation eq. (2.16) for each diagram,

$$
d \mathcal{I}^{j}=\sum_{k} b_{k} d \log f_{1}^{(k)} d \log f_{2}^{(k)} \ldots d \log f_{4 L}^{(k)},
$$

where $f_{i}^{(k)}$ are some functions of external and loop momenta and the coefficients $b_{k}$ are numerical coefficients independent of external kinematics.

In the planar sector, the other two properties (ii) and (iii) are closely related to dual conformal symmetry. As discussed in section 2.1, the exact constraints of dual conformal symmetry on MHV amplitudes are that the amplitudes have unit leading singularities (when combined with ordinary superconformal symmetry and stripped off Parke-Taylor factor) and no poles at infinity. Property (ii) can be directly carried over to any nonplanar integrand, in particular it would imply that the $d$ log forms in eq. (3.2) never generate a pole as $\ell \rightarrow \infty$. As for property (iii), the value of leading singularities cannot be directly translated to the nonplanar case, since there is no single overall Parke-Taylor factor to strip off. Superconformal invariance only allows us to write leading singularities as any holomorphic function $\mathcal{F}_{n}(\lambda)$, but as proven in ref. [56], the only allowed functions are

$$
\mathcal{F}_{n}=\sum_{\sigma} a_{\sigma} \mathrm{PT}_{\sigma}
$$

where $a_{\sigma}=( \pm 1,0)$ and PT stands for a Parke-Taylor factor with a given ordering,

$$
\mathrm{PT}_{\sigma} \equiv \mathrm{PT}\left(\sigma_{1} \sigma_{2} \sigma_{3} \ldots \sigma_{n}\right)=\frac{\delta^{8}(Q)}{\left\langle\sigma_{1} \sigma_{2}\right\rangle\left\langle\sigma_{2} \sigma_{3}\right\rangle \ldots\left\langle\sigma_{n} \sigma_{1}\right\rangle} .
$$

The sum over $\sigma$ runs over the Parke-Taylor amplitudes independent under the Kleiss-Kuijf relations [74]. There are additional relations between the amplitudes, but those introduce ratios of kinematic invariants [50] — which introduce spurious poles in external kinematics since they involve $\tilde{\lambda}$ - and so we will not make use of them here.

As an example, consider the on-shell diagram from figure 4 above, which is equal to the sum of seven Parke-Taylor factors (see eq. (3.11) of ref. [56]),

$$
\begin{aligned}
\mathcal{F}_{6}= & \mathrm{PT}(123456)+\mathrm{PT}(124563)+\mathrm{PT}(142563)+\mathrm{PT}(145623) \\
& +\mathrm{PT}(146235)+\mathrm{PT}(146253)+\mathrm{PT}(162345)
\end{aligned}
$$

This is a nontrivial property since there exist many holomorphic functions $\mathcal{F}_{n}(\lambda)$ for $n \geq 6$ which are not of the form of eq. (3.3).

Analogously to how it works in the planar sector, we can define a pure integrand to take the form eq. (2.16), so that the integrand has unit logarithmic singularities with no poles at infinity. Putting together the results from refs. [52, 53, 56], our conjecture is that all MHV amplitudes in $\mathcal{N}=4$ SYM theory can be written as

$$
\mathcal{M}=\sum_{k, \sigma, j} a_{\sigma, k, j} c_{k} \mathrm{PT}_{\sigma} \int d \mathcal{I}^{j},
$$


where $a_{\sigma, k, j}$ are numerical rational coefficients and $d \mathcal{I}^{j}$ are pure integrands with leading singularities $( \pm 1,0)$. The $\mathrm{PT}_{\sigma}$ are as in eq. (3.4), and $c_{k}$ are color factors. For contributions with the maximum number of propagators, the unique color factors can be read off directly from the corresponding diagrams, but contact term contributions may have multiple contributing color factors. The $a_{\sigma, k, j}$ coefficients are such that, up to sums of Parke-Taylor factors, the leading singularities of the amplitude are normalized to be $( \pm 1,0)$, reflecting a known property of the amplitude.

\subsubsection{Uniqueness and total derivatives}

There is an important question about the uniqueness of our result. The standard wisdom is that the final amplitude $\mathcal{M}$ is a unique object while the planar integrand $d \mathcal{I}$ is ambiguous, as we can add any total derivative $d \mathcal{I}^{\text {tot }}$,

$$
\int d \mathcal{I}^{\text {tot }}=0
$$

that leaves $\mathcal{M}$ invariant. Note that this is not true in our way of constructing the integrand, which relies on matching the cuts of the amplitude. This was sharply stated in ref. [31]: there is only one function which satisfies all constraints (logarithmic singularities, dual conformal symmetry) and cut conditions. Any total derivative $d \mathcal{I}^{\text {tot }}$ would violate one or the other. In other words, if we demand dual conformal invariance and logarithmic singularities then any integrand would necessarily contribute to some of the cuts; the integrand therefore cannot be left undetected by all cuts. It does not matter if it integrates to zero or not, its coefficient is completely fixed by cut conditions.

The same is true in the case of nonplanar amplitudes in general. In practice, our bases of pure integrands for all examples in the following subsections are complete. The pure integrand representation does not distinguish between forms that do integrate to zero and those that do not. Therefore, once the cuts are matched, the pure integrand basis does not miss any total derivatives that satisfy our constraints, and thus we cannot add any terms like $\int d \mathcal{I}^{\text {tot }}$ to our amplitude. In fact, some linear combination of the basis elements $d \mathcal{I}^{j}$ in eq. (3.6) might be total derivatives, but the linear combination must contribute to the amplitude prior to integration with fixed coefficients to match all cuts. There is no freedom to change this coefficient to some other value. As a result, like in the planar sector, the nonplanar result is unique once we impose all constraints.

In the remainder of this section, we explicitly demonstrate that the two-loop fourpoint, three-loop four-point, and two-loop five-point amplitudes may be written in this pure integrand expansion. In section 4 , we furthermore demonstrate that the coefficients $a_{\sigma, k, j}$ are all determined from homogeneous information.

\subsection{Two-loop four-point amplitude}

The simplest multi-loop example is the two-loop four-point amplitude, which was first obtained in refs. [73, 75]. In ref. [52] these results were reorganized in terms of individual integrals with only logarithmic singularities and no poles at infinity. There are two topologies: planar and nonplanar double boxes, as illustrated in figure 5. The numerators for the 


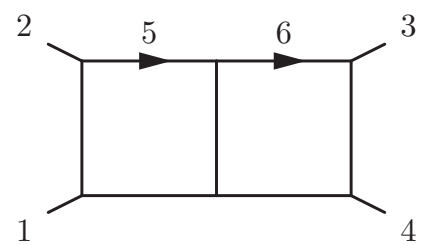

(p)

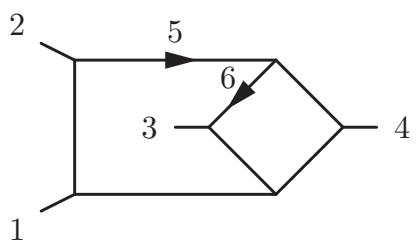

(np)

Figure 5. The integrals appearing in the two-loop four-point amplitude of $\mathcal{N}=4 \mathrm{SYM}$ theory.

planar and nonplanar double box integrals with these properties are

$$
\tilde{N}^{(\mathrm{p})}=s, \quad \tilde{N}^{(\mathrm{np})}=\left(\ell_{5}-k_{3}\right)^{2}+\left(\ell_{5}-k_{4}\right)^{2},
$$

up to overall factors independent of loop momentum, $\ell_{i}$. The labels on the momenta in eq. (3.8) correspond to the leg labels in figure 5. The integrand $d \mathcal{I}^{(\mathrm{np})}$ with this numerator has logarithmic singularities and no poles at infinity, but it is not a pure integrand. That is, the leading singularities are not all \pm 1 but also contain ratios of the form, $\pm u / t$. The kinematic invariants $s=\left(k_{1}+k_{2}\right)^{2}, t=\left(k_{2}+k_{3}\right)^{2}$ and $u=\left(k_{1}+k_{3}\right)^{2}$ are the usual Mandelstam invariants.

Here we want to decompose the $\tilde{N}$ numerators so that the resulting integrands $d \mathcal{I}^{j}$ are pure, and express the amplitude in terms of the resulting pure integrand basis. In practice, we do this by retaining (with respect to ref. [52]) the permutation invariant function $\mathcal{K}=s t \mathrm{PT}(1234)=s u \mathrm{PT}(1243)$, and by requiring each basis integrand to have correct mass dimension - six in this case - and unit leading singularities \pm 1 . This gives us three basis elements:

$$
N^{(\mathrm{p})}=s^{2} t, \quad N_{1}^{(\mathrm{np})}=s u\left(\ell_{5}-k_{3}\right)^{2}, \quad N_{2}^{(\mathrm{np})}=s t\left(\ell_{5}-k_{4}\right)^{2} .
$$

The two nonplanar basis integrals are related by the symmetry of the diagram, but to maintain unit leading singularities we keep the terms distinct. The corresponding pure integrand forms $d \mathcal{I}^{(\mathrm{p})}, d \mathcal{I}_{1}^{(\mathrm{np})}, d \mathcal{I}_{2}^{(\mathrm{np})}$ are obtained by including the integration measure and the appropriate propagators that can be read off from figure 5

We note that for the planar double box, an explicit $d \log$ form is known [52],

$$
\begin{aligned}
d \mathcal{I}^{(\mathrm{p})}= & d \log \frac{\ell_{5}^{2}}{\left(\ell_{5}-\ell_{5}^{*}\right)^{2}} d \log \frac{\left(\ell_{5}+k_{2}\right)^{2}}{\left(\ell_{5}-\ell_{5}^{*}\right)^{2}} d \log \frac{\left(\ell_{5}+k_{1}+k_{2}\right)^{2}}{\left(\ell_{5}-\ell_{5}^{*}\right)^{2}} d \log \frac{\left(\ell_{5}-k_{3}\right)^{2}}{\left(\ell_{5}-\ell_{5}^{*}\right)^{2}} \\
& \times d \log \frac{\left(\ell_{5}-\ell_{6}\right)^{2}}{\left(\ell_{6}-\ell_{6}^{*}\right)^{2}} d \log \frac{\ell_{6}^{2}}{\left(\ell_{6}-\ell_{6}^{*}\right)^{2}} d \log \frac{\left(\ell_{6}-k_{3}\right)^{2}}{\left(\ell_{6}-\ell_{6}^{*}\right)^{2}} d \log \frac{\left(\ell_{6}-k_{3}-k_{4}\right)^{2}}{\left(\ell_{6}-\ell_{6}^{*}\right)^{2}}
\end{aligned}
$$

where

$$
\ell_{5}^{*}=-\frac{\langle 12\rangle}{\langle 13\rangle} \lambda_{3} \widetilde{\lambda}_{2}, \quad \ell_{6}^{*}=k_{3}+\frac{\left(\ell_{5}-k_{3}\right)^{2}}{\left\langle 4\left|\ell_{5}\right| 3\right]} \lambda_{4} \widetilde{\lambda}_{3},
$$

denote one of the solutions to the on-shell conditions. Ref. [52] gave the $d \log$ form for the nonplanar double box with numerators as in eq. (3.9) as a sum of four $d \log$ forms with prefactors (leading to different Parke-Taylor factors). This representation has the 
advantage that it naturally separates parity even and odd pieces. In ref. [53] this was rewritten in a way that manifestly splits into unit leading singularity pieces, so that there are single $d \log$ forms corresponding to each of the nonplanar numerators $N_{1}^{(\mathrm{np})}$ and $N_{2}^{(\mathrm{np})}$. As usual, we suppress the wedge notation and write,

$$
d \mathcal{I}_{1}^{(\mathrm{np})}=d \Omega_{1} d \Omega_{2,(1)}, \quad d \mathcal{I}_{2}^{(\mathrm{np})}=d \Omega_{1} d \Omega_{2,(2)} .
$$

More explicitly, these forms are

$$
\begin{aligned}
d \Omega_{1} & =d \log \frac{\ell_{6}^{2}}{\left(\ell_{6}-\ell_{6}^{*}\right)^{2}} d \log \frac{\left(\ell_{6}-k_{3}\right)^{2}}{\left(\ell_{6}-\ell_{6}^{*}\right)^{2}} d \log \frac{\left(\ell_{6}-\ell_{5}\right)^{2}}{\left(\ell_{6}-\ell_{6}^{*}\right)^{2}} d \log \frac{\left(\ell_{6}-\ell_{5}+k_{4}\right)^{2}}{\left(\ell_{6}-\ell_{6}^{*}\right)^{2}}, \\
d \Omega_{2,(1)} & =d \log \frac{\ell_{5}^{2}}{\left\langle 4\left|\ell_{5}\right| 3\right]} d \log \frac{\left(\ell_{5}+k_{2}\right)^{2}}{\left\langle 4\left|\ell_{5}\right| 3\right]} d \log \frac{\left(\ell_{5}+k_{1}+k_{2}\right)^{2}}{\left\langle 3\left|\ell_{5}\right| 4\right]} d \log \frac{\left(\ell_{5}-\ell_{5,1}^{*}\right)^{2}}{\left\langle 3\left|\ell_{5}\right| 4\right]}, \\
d \Omega_{2,(2)} & =d \log \frac{\ell_{5}^{2}}{\left\langle 3\left|\ell_{5}\right| 4\right]} d \log \frac{\left(\ell_{5}+k_{2}\right)^{2}}{\left\langle 3\left|\ell_{5}\right| 4\right]} d \log \frac{\left(\ell_{5}+k_{1}+k_{2}\right)^{2}}{\left\langle 4\left|\ell_{5}\right| 3\right]} d \log \frac{\left(\ell_{5}-\ell_{5,2}^{*}\right)^{2}}{\left\langle 4\left|\ell_{5}\right| 3\right]} .
\end{aligned}
$$

where the cut solutions read

$$
\ell_{6}^{*}=-\frac{\lambda_{3} \ell_{5} \cdot \lambda_{4}}{\langle 34\rangle}, \quad \ell_{5,1}^{*}=-\frac{\langle 34\rangle}{\langle 31\rangle} \lambda_{1} \widetilde{\lambda}_{4}-k_{1}-k_{2}, \quad \ell_{5,2}^{*}=-\frac{\langle 43\rangle}{\langle 41\rangle} \lambda_{1} \widetilde{\lambda}_{3}-k_{1}-k_{2} .
$$

Using these basis integrals, the full two-loop four-point amplitude can be written as a linear combination dressed with the appropriate color and Parke-Taylor factors,

$$
\begin{aligned}
& \mathcal{M}_{4}^{2 \text {-loop }}=\frac{1}{4} \sum_{S_{4}}\left[c_{1234}^{(\mathrm{p})} a^{(\mathrm{p})} \mathrm{PT}(1234) \int d \mathcal{I}^{(\mathrm{p})}\right. \\
& \left.\quad+c_{1234}^{(\mathrm{np})}\left(a_{1}^{(\mathrm{np})} \mathrm{PT}(1243) \int d \mathcal{I}_{1}^{(\mathrm{np})}+a_{2}^{(\mathrm{np})} \mathrm{PT}(1234) \int d \mathcal{I}_{2}^{(\mathrm{np})}\right)\right]
\end{aligned}
$$

where we sum over all 24 permutations of the external legs $S_{4}$. The overall $1 / 4$ divides out the symmetry factor for each diagram to remove the overcount from the permutation sum. The planar and nonplanar double-box color factors are

$$
\begin{aligned}
& c_{1234}^{(\mathrm{p})}=\tilde{f}^{a_{1} a_{7} a_{9}} \tilde{f}^{a_{2} a_{5} a_{7}} \tilde{f}^{a_{5} a_{6} a_{8}} \tilde{f}^{a_{9} a_{8} a_{10}} \tilde{f}^{a_{3} a_{11} a_{6}} \tilde{f}^{a_{4} a_{10} a_{11}}, \\
& c_{1234}^{(\mathrm{np})}=\tilde{f}^{a_{1} a_{7} a_{8}} \tilde{f}^{a_{2} a_{5} a_{7}} \tilde{f}^{a_{5} a_{11} a_{6}} \tilde{f}^{a_{8} a_{9} a_{10}} \tilde{f}^{a_{3} a_{6} a_{9}} \tilde{f}^{a_{4} a_{10} a_{11}},
\end{aligned}
$$

where the $\tilde{f}^{a b c}=i \sqrt{2} f^{a b c}$ are appropriately normalized color structure constants.

Matching the amplitude on unitarity cuts determines the coefficients to be

$$
a^{(\mathrm{p})}=1, \quad a_{1}^{(\mathrm{np})}=-1, \quad a_{2}^{(\mathrm{np})}=-1,
$$

so that the amplitude in eq. (3.15) is equivalent to the one presented in ref. [52]. The trivial difference is that there the two pieces $d \mathcal{I}_{1}^{(\mathrm{np})}$ and $d \mathcal{I}_{2}^{(\mathrm{np})}$ are combined into one numerator.

\subsection{Three-loop four-point amplitude}

Now consider the three-loop four-point amplitude. This amplitude has been discussed already in various papers $[51,53,58,76]$. Here we will express the amplitude in a pure 
integrand basis. In order to find such a basis we follow the strategy of ref. [53], wherein integrands with only logarithmic singularities were identified. We proceed in the same way, but at the end impose the additional requirement that the leading singularities be \pm 1 or 0 . The construction of diagram numerators which lead to pure integrands is very similar to the previous representation of ref. [53], so we will only summarize the construction here.

The construction starts from a general $\mathcal{N}=4 \mathrm{SYM}$ power counting of loop momenta. For a given loop variable we require the overall scaling of a given integrand to behave like a box in that variable. For example, if there is a pentagon subdiagram for loop variable $\ell$, we allow a nontrivial numerator in $\ell, N \sim \rho_{1} \ell^{2}+\rho_{2}(\ell \cdot Q)+\rho_{3}$, where $Q$ is some complex momentum (not necessarily massless). Similarly, if there is a hexagon subdiagram in loop variable $\ell$, we allow $N \sim \rho_{1}\left(\ell^{2}\right)^{2}+\rho_{2}\left(\ell^{2}\right)(\ell \cdot Q)+\rho_{3}\left(\ell \cdot Q_{1}\right)\left(\ell \cdot Q_{2}\right)+\ldots$, and so on. Our conventions require that the overall mass dimension of $d \mathcal{I}_{j}$ is zero ${ }^{5}$ which fixes the mass dimension of the $\rho_{j}$.

In ref. [53], we then directly constructed the amplitude by constraining the ansatz numerators to obey the symmetry of the diagrams and to vanish on poles at infinity and double (or multiple) poles. We now take a slightly different approach and instead of constructing the amplitude directly, focus on constructing the pure integrand basis.

\subsubsection{Basis of unit leading singularity numerators}

The next step in constructing the pure integrand basis is to require the elements have unit leading singularities. We write each basis element as an ansatz that has the same power counting as the diagram numerators. We then constrain the elements so that any leading singularity - codimension $4 L$ residue - is either \pm 1 or 0 .

The resulting basis elements differ slightly from those of ref. [53]. Terms that were originally grouped so that the numerator obeyed diagram symmetry are now split to make the unit leading singularity property manifest. This is exactly the same reason we rewrote eq. (3.8) as eq. (3.9) in the two-loop four-point example. Additionally, the basis elements are scaled by products $s t, s u$, or $t u$ to account for differing normalizations. The results of our construction of basis numerators yielding pure integrands are summarized in table 1 .

In table 1 we use the relabeling convention $\left.N\right|_{i \leftrightarrow j}$ : "redraw the graph associated with numerator $N$ with the indicated exchanges of external momenta $i, j$ and also relabel loop momenta accordingly." As a simple example look at $\left.N_{1}^{(i)}\right|_{1 \leftrightarrow 3}$,

$$
N_{1}^{(\mathrm{i})}=t u\left(\ell_{6}+k_{4}\right)^{2}\left(\ell_{5}-k_{1}-k_{2}\right)^{2}, \quad N_{2}^{(\mathrm{i})}=\left.N_{1}^{(\mathrm{i})}\right|_{1 \leftrightarrow 3} .
$$

Under this relabeling, the Mandelstam variables $s$ and $t$ transform into one another $s=$ $\left(k_{1}+k_{2}\right)^{2} \leftrightarrow\left(k_{3}+k_{2}\right)^{2}=t$ and $u$ stays invariant. As usual we take the labels of the momenta to follow the labels of the corresponding diagrams. Here it is the labels of diagram (i) in table 1. Besides changing the external labels, we are instructed to relabel the loop momenta as well. In the chosen example, this corresponds to interchanging $\ell_{5} \leftrightarrow \ell_{6}$, so that

$$
N_{2}^{(\mathrm{i})}=\left.N_{1}^{(\mathrm{i})}\right|_{1 \leftrightarrow 3}=\operatorname{su}\left(\ell_{5}+k_{4}\right)^{2}\left(\ell_{6}-k_{3}-k_{2}\right)^{2} .
$$

\footnotetext{
${ }^{5}$ This mass dimension is different than in ref. [53], where we factored out the totally crossing symmetric $\mathcal{K}=s t \mathrm{PT}(1234)=\operatorname{suPT}(1243)=t u \mathrm{PT}(1324)$.
} 


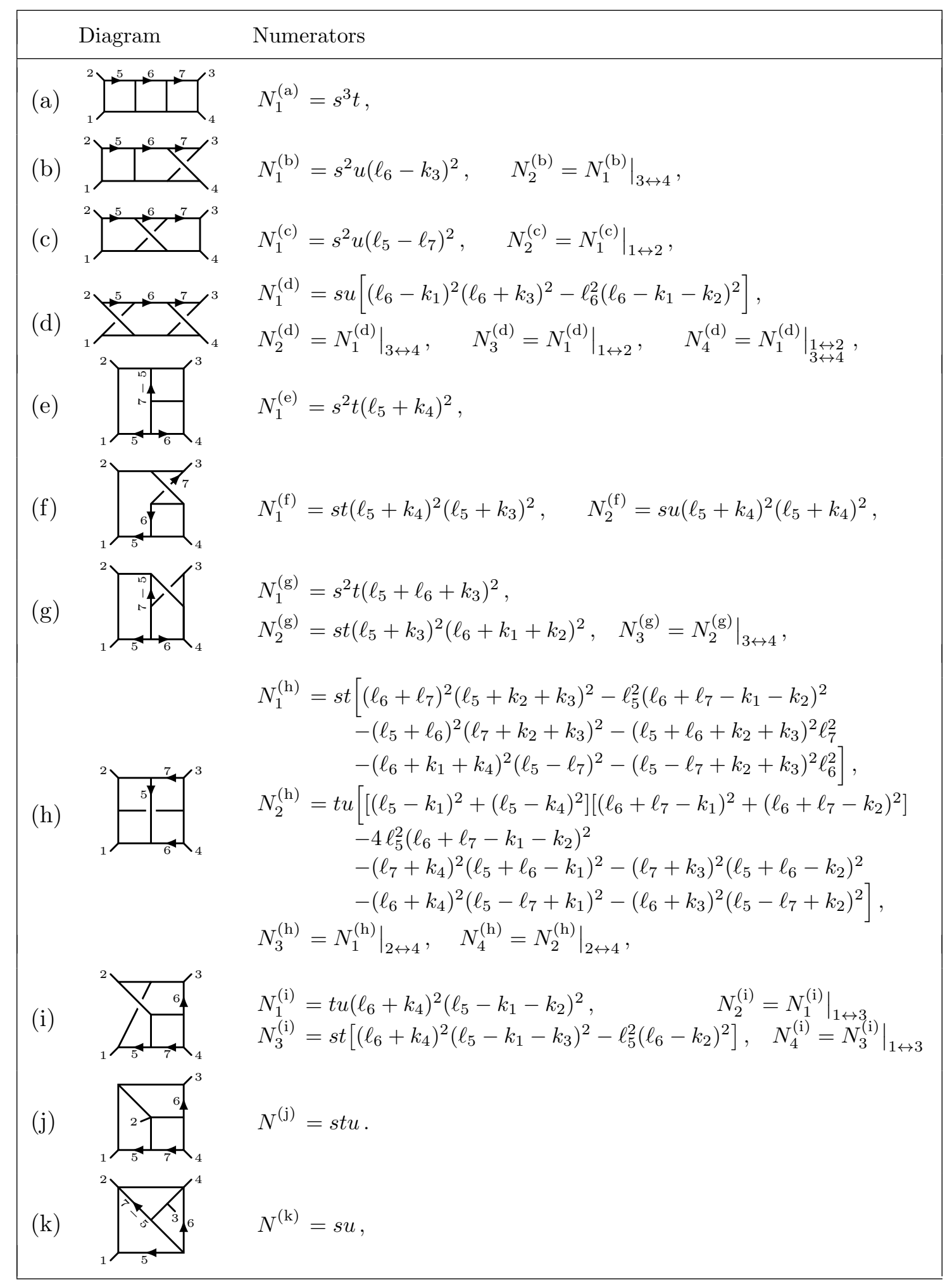

Table 1. The basis of numerators for pure integrands for the three-loop four-point amplitude. The notation $\left.N\right|_{i \leftrightarrow j}$ is defined in the text. 


\subsubsection{Matching the amplitude}

The three-loop four-point amplitude is assembled from the basis numerators as

$$
\mathcal{M}_{4}^{3 \text {-loop }}=\sum_{S_{4}} \sum_{x} \frac{1}{\mathcal{S}_{x}} \int d^{4} \ell_{5} d^{4} \ell_{6} d^{4} \ell_{7} \frac{\mathcal{N}^{(x)}}{\prod_{\alpha_{x}} p_{\alpha_{x}}^{2}},
$$

analogously to eq. (3.15). Now the sum over $x$ runs over all diagrams in the basis listed in table 1 , the sum over $S_{4}$ is a sum over all 24 permutations of the external legs, and $\mathcal{S}_{x}$ is the symmetry factor of diagram $x$ determined by counting the number of automorphisms of diagram $x$. The product over $\alpha_{x}$ indicates the product of Feynman propagators $p_{\alpha_{x}}^{2}$ of diagram $x$, as read from the graphs in table 1 . The Parke-Taylor factors, color factors, and coefficients are absorbed in $\mathcal{N}^{(x)}$, which we list in table 2 .

For four external particles, there are only two independent Parke-Taylor factors. We abbreviate these as

$$
\mathrm{PT}_{1}=\mathrm{PT}(1234), \quad \mathrm{PT}_{2}=\mathrm{PT}(1243) .
$$

The third possible factor, $\mathrm{PT}(1423)$, is related to the other two by a U(1) decoupling identity or dual Ward identity [77]

$$
\mathrm{PT}(1423)=-\mathrm{PT}(1234)-\mathrm{PT}(1243)
$$

and is therefore linearly dependent on $\mathrm{PT}_{1}$ and $\mathrm{PT}_{2}$.

When checking cuts of the amplitude, certain cuts may combine contributions from different terms in the permutation sum of eq. (3.20), resulting in a cut expression that involves diagrams that are relabellings of those in table 1 . In that case, the procedure is to relabel the numerators, propagators, Parke-Taylor factors, and color factors given in the tables into the cut labels. The resulting Parke-Taylor factors may not be in the original basis of Parke-Taylor factors; however every Parke-Taylor in the relabeled expression can be expanded in the original Parke-Taylor basis.

The diagrams with 10 propagators contain only three-point vertices and therefore have unique color factors included in $\mathcal{N}^{(x)}$. For the two diagrams with less than 10 propagators, we include in our ansatz for $\mathcal{N}$ all independent color factors from all 10-propagator diagrams that are related to the lower-propagator diagrams by collapsing internal legs. For example, three 10-propagator diagrams are related to diagram (j) in this way, with color factors $c_{1234}^{(\mathrm{i})}, c_{1243}^{(\mathrm{i})}$ and $c_{3241}^{(\mathrm{i})}$, where

$$
c_{1234}^{(\mathrm{i})}=\tilde{f}^{a_{1} a_{8} a_{5}} \tilde{f}^{a_{6} a_{2} a_{9}} \tilde{f}^{a_{3} a_{11} a_{10}} \tilde{f}^{a_{12} a_{4} a_{13}} \tilde{f}^{a_{9} a_{10} a_{8}} \tilde{f}^{a_{11} a_{12} a_{14}} \tilde{f}^{a_{13} a_{5} a_{7}} \tilde{f}^{a_{14} a_{7} a_{6}},
$$

is the standard color factor in terms of appropriately normalized structure constants, and the others $c$ 's are relabellings of 1234 of this color factor. The Jacobi relation between the three color factors allows us to eliminate, say $c_{1243}^{(\mathrm{i})}$. This is exactly what we do for diagram (j). In diagram $(\mathrm{k})$, there are nine contributing parent diagrams. Typically there are four independent color factors in the solution to the set of six Jacobi relations, but in this case two of the color factors that contribute happen to be identical up to a sign, and thus there are only three independent color factors. 


\begin{tabular}{|c|c|}
\hline Color Dressed Numerators & PT Matrices \\
\hline $\mathcal{N}^{(\mathrm{a})}=c_{1234}^{(\mathrm{a})} \sum_{1 \leq \sigma \leq 2} N_{1}^{(\mathrm{a})} \mathrm{a}_{1 \sigma}^{(\mathrm{a})} \mathrm{PT}_{\sigma}$ & $a_{1 \sigma}^{(a)}=\left(\begin{array}{ll}1 & 0\end{array}\right)$, \\
\hline $\mathcal{N}^{(\mathrm{b})}=c_{1234}^{(\mathrm{b})} \sum_{\substack{1 \leq \nu \leq 2 \\
1 \leq \sigma \leq 2}} N_{\nu}^{(\mathrm{b})} \mathrm{a}_{\nu \sigma}^{(\mathrm{b})} \mathrm{PT}_{\sigma}$ & $\mathrm{a}_{\nu \sigma}^{(\mathrm{b})}=(-1)\left(\begin{array}{ll}0 & 1 \\
1 & 0\end{array}\right)$ \\
\hline $\mathcal{N}^{(\mathrm{c})}=c_{1234}^{(\mathrm{c})} \sum_{\substack{1 \leq \nu \leq 2 \\
1 \leq \sigma \leq 2}} N_{\nu}^{(\mathrm{c})} \mathrm{a}_{\nu \sigma}^{(\mathrm{c})} \mathrm{PT}_{\sigma}$ & $a_{\nu \sigma}^{(\mathrm{c})}=(-1)\left(\begin{array}{ll}0 & 1 \\
1 & 0\end{array}\right)$ \\
\hline $\mathcal{N}^{(\mathrm{d})}=c_{1234}^{(\mathrm{d})} \sum_{\substack{1 \leq \nu \leq 4 \\
1<\sigma<2}} N_{\nu}^{(\mathrm{d})} \mathrm{a}_{\nu \sigma}^{(\mathrm{d})} \mathrm{PT}_{\sigma}$ & $\mathrm{a}_{\nu \sigma}^{(\mathrm{d})}=\left(\begin{array}{llll}0 & 1 & 1 & 0 \\
1 & 0 & 0 & 1\end{array}\right)^{T}$ \\
\hline $\mathcal{N}^{(\mathrm{e})}=c_{1234}^{(\mathrm{e})} \sum_{1 \leq \sigma \leq 2} N_{1}^{(\mathrm{e})} \mathrm{a}_{1 \sigma}^{(\mathrm{e})} \mathrm{PT}_{\sigma}$ & $a_{1 \sigma}^{(e)}=\left(\begin{array}{ll}1 & 0\end{array}\right)$, \\
\hline $\mathcal{N}^{(\mathrm{f})}=c_{1234}^{(\mathrm{f})} \sum_{\substack{1 \leq \nu \leq 2 \\
1 \leq \sigma \leq 2}} N_{\nu}^{(\mathrm{f})} \mathrm{a}_{\nu \sigma}^{(\mathrm{f})} \mathrm{PT}_{\sigma}$ & $a_{\nu \sigma}^{(f)}=(-1)\left(\begin{array}{ll}1 & 0 \\
0 & 1\end{array}\right)$ \\
\hline $\mathcal{N}^{(\mathrm{g})}=c_{1234}^{(\mathrm{g})} \sum_{\substack{1 \leq \nu \leq 3 \\
1 \leq \sigma \leq 2}} N_{\nu}^{(\mathrm{g})} \mathrm{a}_{\nu \sigma}^{(\mathrm{g})} \mathrm{PT}_{\sigma}$ & $\mathrm{a}_{\nu \sigma}^{(\mathrm{g})}=\left(\begin{array}{ccc}-1 & 1 & 0 \\
0 & 0 & 1\end{array}\right)^{T}$ \\
\hline $\mathcal{N}^{(\mathrm{h})}=c_{1234}^{(\mathrm{h})} \sum_{\substack{1 \leq \nu \leq 4 \\
1 \leq \sigma \leq 2}} N_{\nu}^{(\mathrm{h})} \mathrm{a}_{\nu \sigma}^{(\mathrm{h})} \mathrm{PT}_{\sigma}$ & $\mathrm{a}_{\nu \sigma}^{(\mathrm{h})}=\frac{1}{2}\left(\begin{array}{cccc}1 & 1 & 1 & 0 \\
0 & 1 & 0 & -1\end{array}\right)^{T}$ \\
\hline $\mathcal{N}^{(\mathrm{i})}=c_{1234}^{(\mathrm{i})} \sum_{\substack{1 \leq \nu \leq 4 \\
1 \leq \sigma \leq 2}} N_{\nu}^{(\mathrm{i})} \mathrm{a}_{\nu \sigma}^{(\mathrm{i})} \mathrm{PT}_{\sigma}$ & $\mathrm{a}_{\nu \sigma}^{(\mathrm{i})}=(-1)\left(\begin{array}{cccc}1 & 0 & -1 & 1 \\
1 & 1 & 0 & 0\end{array}\right)^{T}$ \\
\hline $\mathcal{N}^{(\mathrm{j})}=c_{1234}^{(\mathrm{i})} \sum_{1 \leq \sigma \leq 2} N_{1}^{(\mathrm{j})} \mathrm{a}_{1 \sigma,(1234)}^{(\mathrm{j})} \mathrm{PT}_{\sigma}$ & $\mathrm{a}_{1 \sigma,(1234)}^{(\mathrm{j})}=\left(\begin{array}{ll}1 & 1\end{array}\right)$, \\
\hline$+c_{3241}^{(\mathrm{i})} \sum_{1 \leq \sigma \leq 2} N_{1}^{(\mathrm{j})} \mathrm{a}_{1 \sigma,(3241)}^{(\mathrm{j})} \mathrm{PT}_{\sigma}$ & $\mathrm{a}_{1 \sigma,(3241)}^{(\mathrm{j})}=\left(\begin{array}{ll}-1 & 0\end{array}\right)$, \\
\hline $\mathcal{N}^{(\mathrm{k})}=c_{1234}^{(\mathrm{g})} \sum_{1 \leq \sigma \leq 2} N_{1}^{(\mathrm{k})} \mathrm{a}_{1 \sigma,(1234)}^{(\mathrm{k})} \mathrm{PT}_{\sigma}$ & $\mathrm{a}_{1 \sigma,(1234)}^{(\mathrm{k})}=\left(\begin{array}{ll}-2 & 0\end{array}\right)$ \\
\hline$+c_{4312}^{(\mathrm{g})} \sum_{1 \leq \sigma \leq 2} N_{1}^{(\mathrm{k})} \mathrm{a}_{1 \sigma,(4312)}^{(\mathrm{k})} \mathrm{PT}_{\sigma}$ & $\mathrm{a}_{1 \sigma,(4312)}^{(\mathrm{k})}=0$ \\
\hline$+c_{2431}^{(\mathrm{f})} \sum_{1 \leq \sigma \leq 2} N_{1}^{(\mathrm{k})} \mathrm{a}_{1 \sigma,(2431)}^{(\mathrm{k})} \mathrm{PT}_{\sigma}$. & $\mathrm{a}_{1 \sigma,(2431)}^{(\mathrm{k})}=0$. \\
\hline
\end{tabular}

Table 2. The three-loop four-point numerators that contribute to the amplitude. The $N_{\nu}^{(\mathrm{x})}$ are listed in table 1. The four-point Parke-Taylor factors $\mathrm{PT}_{\sigma}$ are listed in eq. (3.21). The numerators including color factors are denoted as $\mathcal{N}^{(x)}$. The symbol ' $T$ ' denotes a transpose. 
In ref. [53], the final representation of the amplitude contained arbitrary free parameters associated with the color Jacobi identity that allowed contact terms to be moved between parent diagrams without altering the amplitude. Here we removed this freedom by assigning the contact terms to their own diagrams and keeping only a basis of independent color factors for each.

One advantage of the Parke-Taylor expansion of the amplitude is that we can compactly express the solution to the cut equations in the set of matrices listed on the right hand side of table 2. For example, $\mathcal{N}^{(i)}$ can be read off from the table as

$$
\mathcal{N}^{(\mathrm{i})}=c_{1234}^{(\mathrm{i})}(-1)\left(N_{1}^{(\mathrm{i})}\left(\mathrm{PT}_{1}+\mathrm{PT}_{2}\right)+N_{2}^{(\mathrm{i})} \mathrm{PT}_{2}-N_{3}^{(\mathrm{i})} \mathrm{PT}_{1}+N_{4}^{(\mathrm{i})} \mathrm{PT}_{1}\right)
$$

This expression supplies the Parke-Taylor and color dependence required for eq. (3.20), in agreement with the general form of eq. (3.6).

\subsection{Two-loop five-point amplitude}

The integrand for the two-loop five-point amplitude was first obtained in ref. [59] in a format that makes the duality between color and kinematics manifest. Here we find a pure integrand representation. An additional feature of our representation is that it is manifestly free of spurious poles in external kinematics.

\subsubsection{Basis of unit leading-singularity numerators}

Following the three-loop four-point case, our first step is to construct a pure integrand basis. Constructing this basis is similar to constructing the three-loop four-point amplitude in ref. [53] and summarized in section 3.3. Although deriving the numerators for the two-loop five-point case is in principle straightforward, it does require a nontrivial amount of algebra, which we suppress. We again split the basis elements according to diagram topologies and distinguish between parent diagrams and contact diagrams. The numerators of each pure integrand are given in table 3.

Table 4 contains an additional pure integrand. However we do not include it in our basis because it is linearly dependent on two other basis elements: $N_{1}^{(\mathrm{h})}-N_{2}^{(\mathrm{h})}+N_{1}^{(\mathrm{j})}\left(\ell_{6}-k_{1}\right)^{2}=0$. In our result, we choose $N_{1}^{(\mathrm{h})}$ and $N_{2}^{(\mathrm{h})}$ as our linearly independent pure integrands, and only mention $N_{1}^{(\mathrm{j})}$ because it might be an interesting object in future studies.

In contrast to the three-loop four-point basis, in the two-loop five-point case it is useful to allow spinor helicity variables associated with external momenta. Specifically, several of the expressions in table 3 have the structure $\left(\ell+\alpha \lambda_{i} \widetilde{\lambda}_{j}\right)^{2}$, where $\alpha$ is such that both mass dimension and little group weights are consistent. For example, the penta-box numerator

$$
N_{1}^{(\mathrm{b})} \sim\left(\ell_{6}+\frac{Q_{45} \cdot \tilde{\lambda}_{3} \tilde{\lambda}_{1}}{[13]}\right)^{2}=\left(\ell_{6}-\ell_{6}^{*}\right)^{2},
$$

is a "chiral" numerator that manifestly vanishes on the $\overline{\mathrm{MHV}}$ solution $\ell_{6}=\ell_{6}^{*}[69]$. As a shorthand notation, we use $Q_{i j}=k_{i}+k_{j}$ and $Q_{i j} \cdot \widetilde{\lambda}_{k}=[i k] \lambda_{i}+[j k] \lambda_{j}$. 


$N_{1}$ Numerators
$N_{1}^{(\mathrm{a})}=\langle 13\rangle\langle 24\rangle\left[[24][13]\left(\ell_{7}+\frac{[45]}{[24]} \lambda_{5} \widetilde{\lambda}_{2}\right)^{2}\left(\ell_{6}-\frac{Q_{12} \tilde{\lambda}_{3} \tilde{\lambda}_{1}}{[13]}\right)^{2}\right.$
$N_{2}^{(\mathrm{a})}=\left.N_{1}^{(\mathrm{a})}\right|_{1 \leftrightarrow 2}, \quad N_{4}^{(\mathrm{a})}=\left.N_{1}^{(\mathrm{a})}\right|_{2 \leftrightarrow 4}, \quad N_{1}^{(\mathrm{a})}=\left.N_{1}^{(\mathrm{a})}\right|_{1 \leftrightarrow 4}$
$N_{5}^{(\mathrm{a})}=\bar{N}_{1}^{(\mathrm{a})}, \quad N_{6}^{(\mathrm{a})}=\bar{N}_{2}^{(\mathrm{a})}, \quad N_{7}^{(\mathrm{a})}=\bar{N}_{3}^{(\mathrm{a})}, \quad N_{8}^{(\mathrm{a})}=\bar{N}_{4}^{(\mathrm{a})}$

Table 3. The parent diagram numerators that give pure integrands for the two-loop five-point amplitude. Each basis diagram is consistent with requiring logarithmic singularities and no poles at infinity. The overline notation means $[\cdot] \leftrightarrow\langle\cdot\rangle$ and $Q_{i j} \cdot \widetilde{\lambda}_{k}=[i k] \lambda_{i}+[j k] \lambda_{j}$. As usual, the momentum labels match the diagram labels. 


\begin{tabular}{|l|}
\hline \multicolumn{1}{|c|}{ Diagram } \\
\hline (j) ${ }_{1}^{2} N_{6}^{(\mathrm{j})}=s_{12} s_{35}=\left(N_{2}^{(\mathrm{h})}-N_{1}^{(\mathrm{h})}\right) /\left(\ell_{6}-k_{1}\right)^{2}$, \\
\hline
\end{tabular}

Table 4. A diagram and numerator that gives a pure integrand. However, as indicated in the table and explained in the text, it is not an independent basis element. As usual, the momentum labels match the diagram labels.

\subsubsection{Matching the amplitude}

Following the construction of the pure integrand basis in section 3.4.1 we are ready to build up the amplitude. In complete analogy to eq. (3.20), the two-loop five-point amplitude is assembled from the basis numerators as,

$$
\mathcal{M}_{5}^{2 \text { loop }}=\sum_{S_{5}} \sum_{x} \frac{1}{\mathcal{S}_{x}} \int d^{4} \ell_{6} d^{4} \ell_{7} \frac{\mathcal{N}^{(x)}}{\prod_{\alpha_{x}} p_{\alpha_{x}}^{2}},
$$

where the sum over $x$ runs over all diagrams in the basis listed in table 3 , the sum over $S_{5}$ is a sum over all 120 permutations of the external legs, and $\mathcal{S}_{x}$ is the symmetry factor of diagram $x$. The product over $\alpha_{x}$ indicates the product of Feynman propagators $p_{\alpha_{x}}^{2}$ of diagram $x$, as read from the graphs in table 3 .

We refer the reader to the discussion in section 3.3.2 for explicit examples on how to read table 5. We choose the following set of independent five-point Parke-Taylor basis elements:

$$
\begin{array}{lll}
\mathrm{PT}_{1}=\mathrm{PT}(12345), & \mathrm{PT}_{2}=\mathrm{PT}(12354), & \mathrm{PT}_{3}=\mathrm{PT}(12453), \\
\mathrm{PT}_{4}=\mathrm{PT}(12534), & \mathrm{PT}_{5}=\mathrm{PT}(13425), & \mathrm{PT}_{6}=\mathrm{PT}(15423) .
\end{array}
$$

The basis elements $\bar{N}^{(x)}$ in table 5 do not contribute to the MHV amplitude so those data are omitted from the $\mathbf{a}_{\nu \sigma}^{(x)}$.

\section{Zeros of the integrand}

In the previous section we gave explicit examples of the expansion of the amplitude, eq. (3.1), in terms of a basis of pure integrands, giving new nontrivial evidence that the analytic consequences of dual conformal symmetry hold beyond the planar sector. In this section we take a further step and present evidence that the amplituhedron concept, which is a complete and self-contained geometric definition of the integrand, may exist beyond the planar sector as well.

As already mentioned in previous sections, beyond the planar limit we currently have no alternative other than to use diagrams representing local integrals, eq. (3.1), as a starting point for defining nonplanar integrands. The lack of global variables makes it unclear how to directly test for a geometric construction analogous to the amplituhedron in the nonplanar 


\begin{tabular}{|c|c|}
\hline Color Dressed Numerators & PT Matrices \\
\hline $\mathcal{N}^{(\mathrm{a})}=c_{12345}^{(\mathrm{a})} \sum_{\substack{1 \leq \nu \leq 4 \\
1 \leq \sigma \leq 6}} N_{\nu}^{(\mathrm{a})} \mathrm{a}_{\nu \sigma}^{(\mathrm{a})} \mathrm{PT}_{\sigma}$ & $\mathrm{a}_{\nu \sigma}^{(\mathrm{a})}=\frac{1}{4}\left(\begin{array}{cccccc}-1 & 0 & 1 & 0 & 0 & 2 \\
1 & 0 & -1 & 0 & 0 & 2 \\
-3 & 0 & -1 & 0 & 0 & 2 \\
-1 & 0 & -3 & 0 & 0 & 2\end{array}\right)$ \\
\hline $\mathcal{N}^{(\mathrm{b})}=c_{12345}^{(\mathrm{b})} \sum_{1 \leq \sigma \leq 6} N_{1}^{(\mathrm{b})} \mathrm{a}_{1 \sigma}^{(\mathrm{b})} \mathrm{PT}_{\sigma}$ & $a_{1 \nu}^{(b)}=\left(\begin{array}{llllll}-1 & 0 & 0 & 0 & 0 & 0\end{array}\right)$ \\
\hline $\mathcal{N}^{(\mathrm{c})}=c_{12345}^{(\mathrm{c})} \sum_{\substack{1 \leq \nu \leq 2 \\
1 \leq \sigma \leq 6}} N_{\nu}^{(\mathrm{c})} \mathrm{a}_{\nu \sigma}^{(\mathrm{c})} \mathrm{PT}_{\sigma}$ & $\mathrm{a}_{\nu \sigma}^{(\mathrm{c})}=\left(\begin{array}{cccccc}-1 & 0 & 0 & 0 & 0 & 0 \\
0 & -1 & 0 & 0 & 0 & 0\end{array}\right)$, \\
\hline \multicolumn{2}{|l|}{$\mathcal{N}^{(\mathrm{d})}=\mathcal{N}^{(\mathrm{e})}=\mathcal{N}^{(\mathrm{f})}=0}$, \\
\hline $\mathcal{N}^{(\mathrm{g})}=c_{12345}^{(\mathrm{a})} \sum_{1 \leq \sigma \leq 6} N_{1}^{(\mathrm{g})} \mathrm{a}_{1 \sigma,(12345)}^{(\mathrm{g})} \mathrm{PT}_{\sigma}$ & $a_{1 \sigma,(12345)}^{(\mathrm{g})}=\frac{1}{4}\left(\begin{array}{lllllll}1 & 0 & 3 & 0 & 0 & -2\end{array}\right)$, \\
\hline$+c_{31245}^{(\mathrm{b})} \sum_{1 \leq \sigma \leq 6} N_{1}^{(\mathrm{g})} \mathrm{a}_{1 \sigma,(31245)}^{(\mathrm{g})} \mathrm{PT}_{\sigma}$ & $a_{1 \sigma,(31245)}^{(\mathrm{g})}=\left(\begin{array}{llllll}0 & 0 & -1 & 0 & 0 & 0\end{array}\right)$, \\
\hline $\mathcal{N}^{(\mathrm{h})}=c_{12345}^{(\mathrm{a})} \sum_{\substack{1 \leq \nu \leq 4 \\
1 \leq \sigma \leq 6}} N_{\nu}^{(\mathrm{h})} \mathrm{a}_{\nu \sigma,(12345)}^{(\mathrm{h})} \mathrm{PT}_{\sigma}$ & $\mathrm{a}_{\nu \sigma,(12345)}^{(\mathrm{h})}=\frac{1}{4}\left(\begin{array}{cccccc}2 & 0 & 3 & 0 & 1 & -2 \\
-2 & 0 & -3 & 0 & -1 & 2 \\
4 & 0 & 4 & 0 & 0 & -4\end{array}\right)$ \\
\hline$+c_{12543}^{(\mathrm{a})} \sum_{\substack{1 \leq \nu \leq 4 \\
1 \leq \sigma \leq 6}} N_{\nu}^{(\mathrm{h})} \mathrm{a}_{\nu \sigma,(12543)}^{(\mathrm{h})} \mathrm{PT}_{\sigma}$ & $\mathrm{a}_{\nu \sigma,(12543)}^{(\mathrm{h})}=\mathrm{a}_{\nu \sigma,(12345)}^{(\mathrm{h})}$ \\
\hline $\mathcal{N}^{(\mathrm{i})}=c_{12345}^{(\mathrm{a})} \sum_{1 \leq \sigma \leq 6} N_{1}^{(\mathrm{i})} \mathrm{a}_{1 \sigma,(12345)}^{(\mathrm{i})} \mathrm{PT}_{\sigma}$ & $a_{1 \sigma,(12345)}^{(i)}=2\left(\begin{array}{llllll}0 & 0 & -1 & 0 & 0 & 1\end{array}\right)$ \\
\hline$+c_{13245}^{(\mathrm{a})} \sum_{1 \leq \sigma \leq 6} N_{1}^{(\mathrm{i})} \mathrm{a}_{1 \sigma,(13245)}^{(\mathrm{i})} \mathrm{PT}_{\sigma}$ & $a_{1 \sigma,(13245)}^{(\mathrm{i})}=2\left(\begin{array}{llllll}0 & 0 & 0 & 0 & 0 & 1\end{array}\right)$, \\
\hline$+c_{12543}^{(\mathrm{a})} \sum_{1 \leq \sigma \leq 6} N_{1}^{(\mathrm{i})} \mathrm{a}_{1 \sigma,(12543)}^{(\mathrm{i})} \mathrm{PT}_{\sigma}$ & $a_{1 \sigma,(12543)}^{(\mathrm{i})}=2\left(\begin{array}{llllll}1 & 0 & 1 & 0 & 1 & -1\end{array}\right)$ \\
\hline$+c_{15243}^{(\mathrm{a})} \sum_{1 \leq \sigma \leq 6} N_{1}^{(\mathrm{i})} \mathrm{a}_{1 \sigma,(15243)}^{(\mathrm{i})} \mathrm{PT}_{\sigma}$ & $a_{1 \sigma,(15243)}^{(\mathrm{i})}=2\left(\begin{array}{llllll}0 & 0 & 0 & 0 & -1 & 0\end{array}\right)$. \\
\hline
\end{tabular}

Table 5. The two-loop five-point numerators that contribute to the amplitude. The $N_{\nu}^{(x)}$ are listed in table 3. The five-point $\mathrm{PT}_{\sigma}$ are listed in eq. (3.27). We denote the numerators including color information as $\mathcal{N}^{(x)}$. 


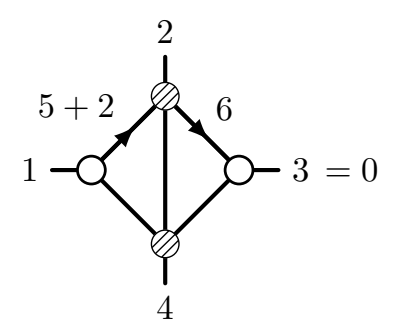

Figure 6. The two-loop four-point MHV amplitude vanishes on this cut. The four-point trees in the diagram have $k=2$, so the overall helicity counting is $k=1$.

sector. However, as discussed in section 2, in the planar sector the (dual) amplituhedron construction implies that all coefficients in the expansion in eq. (2.17) are determined by zero conditions, up to an overall normalization. We expect that if an analogous geometric construction exists in the nonplanar sector, then zero conditions should also determine the amplitude. This can be tested directly. Indeed, we conjecture that for the representation in eq. (3.1):

All coefficients $d_{j}$ are fixed by zero conditions, up to overall normalization.

This is the direct analog of the corresponding planar statement in section 2. In the MHV case, which we consider here, the coefficients $d_{j}$ are linear combinations of Parke-Taylor factors, so that only numerical coefficients $a_{\sigma, k, j}$ in eq. (3.6) need to be determined. The above conjecture is a statement that we can obtain these coefficients using only zero conditions, up to an overall constant. Here we confirm this proposal for all amplitudes constructed in the previous section.

As a simple first example, consider the two-loop four-point amplitude. The integrand is given as a linear combination of planar and nonplanar double boxes, cf. section 3.2. The only required condition to determine the unknown conditions is the cut in figure 6 .

In the full amplitude, we have contributions from the planar and nonplanar double boxes in figure 5 and their permutations of external legs. All permutations of diagrams that contribute to the cut in figure 6 are shown in figure 7 , along with their numerators and color factors. For convenience, we indicate the permutation labels of external legs of the seven contributing diagrams. There are only seven diagrams rather than nine because two of the nine diagrams have triangle subdiagrams, and so have vanishing numerators in $\mathcal{N}=4 \mathrm{SYM}$.

For the cut in figure 6, five propagators are put on-shell so that the cut solution depends on $\alpha, \gamma$, and $\delta$, three unfixed parameters of the loop momenta. Explicitly, the cut solution is

$$
\begin{aligned}
\ell_{5}^{*}+k_{2} & =\lambda_{1}\left[\alpha \widetilde{\lambda}_{1}+\frac{1}{\delta\langle 13\rangle[23]}(\delta t-\alpha(s+\delta u+\gamma\langle 13\rangle[12])) \widetilde{\lambda}_{2}\right], \\
\ell_{6}^{*} & =\lambda_{3}\left[\delta \widetilde{\lambda}_{3}+\gamma \widetilde{\lambda}_{2}\right] .
\end{aligned}
$$




$$
\begin{aligned}
\left.\mathcal{M}_{4}^{2-\text { loop }}\right|_{\text {cut }}= & N_{1234}^{(\mathrm{p})} c_{1234}^{(\mathrm{p})} \\
& +N_{1234}^{(\mathrm{np})} c_{1234}^{(\mathrm{np})} \\
& \\
& +N_{3214}^{(\mathrm{np})} c_{3214}^{(\mathrm{np})} \\
& \\
& +N_{4213}^{(\mathrm{np})} c_{4213}^{(\mathrm{np})}
\end{aligned}
$$$$
\varlimsup_{1}^{2}{ }_{4}^{3}+N_{4123}^{(\mathrm{p})} c_{4123}^{(\mathrm{p})}
$$

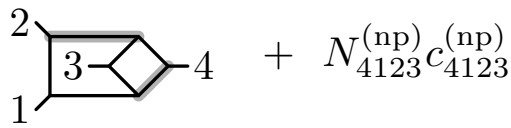$$
\stackrel{2}{1 \preceq 4}+N_{3412}^{(\mathrm{np})} c_{3412}^{(\mathrm{np})}
$$
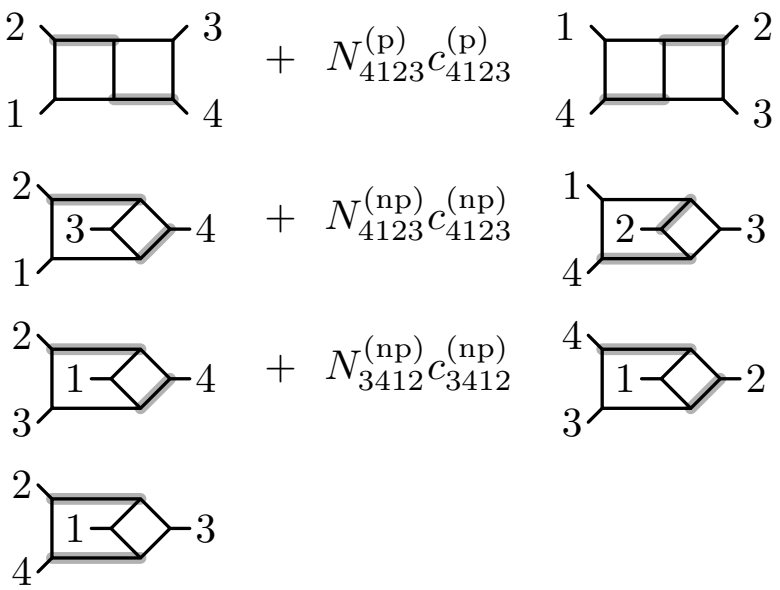

Figure 7. The two-loop four-point amplitude evaluated on the cut of figure 6 . In each diagram the two shaded propagators are uncut, and every other propagator is cut. Eq. (4.7) gives the value of the cut.

On this $k=1$ cut, the MHV amplitude vanishes for any values of $\alpha, \gamma, \delta$. By cutting the Jacobian

$$
J=\gamma(\delta t-\alpha(s+\delta u+\gamma\langle 13\rangle[12]))
$$

the amplitude remains zero, and this condition simplifies. Specifically, this allows us to localize $\ell_{5}+k_{2}$ to be collinear with $k_{1}$ and to localize $\ell_{6}$ to be collinear with $k_{3}$. This is equivalent to taking further residues of the already-cut integrand at $\gamma=0, \alpha=\delta t /(s+\delta u)$. On this cut, the solution for the loop momenta simplifies,

$$
\ell_{5}^{*}+k_{2}=\frac{\delta t}{s+\delta u} \lambda_{1} \widetilde{\lambda}_{1}, \quad \ell_{6}^{*}=\delta \lambda_{3} \widetilde{\lambda}_{3}
$$

with the overall Jacobian $J^{\prime}=s+u \delta$. Even in this simplified setting with one parameter $\delta$ left, the single zero cut condition figure 6 is sufficient to fix the integrand up to an overall constant.

The numerators for the pure integrands, using the labels in figure 5, are given in eq. (3.9). Including labels for the external legs to help us track relabellings, these are

$$
N_{1234}^{(\mathrm{p}, 1)}=s^{2} t, \quad N_{1234}^{(\mathrm{np}, 1)}=s u\left(\ell_{5}-k_{3}\right)^{2}, \quad N_{1234}^{(\mathrm{np}, 2)}=s t\left(\ell_{5}-k_{4}\right)^{2} .
$$

As noted near eq. (3.21) there are only two Parke-Taylor factors independent under U(1) relations for four-particle scattering, namely $\mathrm{PT}_{1}=\mathrm{PT}(1234)$ and $\mathrm{PT}_{2}=\mathrm{PT}(1243)$. Therefore the numerator ansatz for the planar diagram is

$$
N_{1234}^{(\mathrm{p})}=\left(a_{1,1}^{(\mathrm{p})} \mathrm{PT}_{1}+a_{1,2}^{(\mathrm{p})} \mathrm{PT}_{2}\right) N_{1234}^{(\mathrm{p}, 1)}
$$


For the nonplanar diagram, there are two pure integrands, each of which gets decorated with the two independent Parke-Taylor factors, so that the ansatz takes the form

$$
\begin{aligned}
& N_{1234}^{(\mathrm{np})}=\left[\left(a_{1,1}^{(\mathrm{np})} \mathrm{PT}_{1}+a_{1,2}^{(\mathrm{np})} \mathrm{PT}_{2}\right) N_{1234}^{(\mathrm{np}, 1)}\right. \\
& \left.\quad+\left(a_{2,1}^{(\mathrm{np})} \mathrm{PT}_{1}+a_{2,2}^{(\mathrm{np})} \mathrm{PT}_{2}\right) N_{1234}^{(\mathrm{np}, 2)}\right]
\end{aligned}
$$

and both numerators are then decorated with corresponding color factors $c_{1234}^{(\mathrm{p})}, c_{1234}^{(\mathrm{np})}$ and propagators. The $a_{i, j}^{(x)}$ coefficients are determined by demanding the integrand vanishes on the cut solution in eq. (4.3).

Explicitly, the zero condition from the cut corresponding to figure 7 is:

$$
\begin{aligned}
0=\left(\frac{c_{1234}^{(\mathrm{p})} N_{1234}^{(\mathrm{p})}}{\ell_{5}^{2}\left(\ell_{6}-k_{3}-k_{4}\right)^{2}}+\frac{c_{4123}^{(\mathrm{p})} N_{4123}^{(\mathrm{p})}}{\left(\ell_{5}-k_{3}\right)^{2}\left(\ell_{6}+k_{2}\right)^{2}}+\frac{c_{1234}^{(\mathrm{np})} N_{1234}^{(\mathrm{np})}}{\ell_{5}^{2}\left(\ell_{5}-\ell_{6}-k_{4}\right)^{2}}\right. \\
\quad+\frac{c_{4123}^{(\mathrm{np})} N_{4123}^{(\mathrm{np})}}{\left(\ell_{5}-k_{3}\right)^{2}\left(\ell_{5}-\ell_{6}+k_{2}\right)^{2}}+\frac{c_{3214}^{(\mathrm{np})} N_{3214}^{(\mathrm{np})}}{\left(\ell_{6}+k_{2}\right)^{2}\left(\ell_{5}-\ell_{6}-k_{4}\right)^{2}} \\
\left.\quad+\frac{c_{3412}^{(\mathrm{np})} N_{3412}^{(\mathrm{np})}}{\left(\ell_{6}-k_{3}-k_{4}\right)^{2}\left(\ell_{5}-\ell_{6}+k_{2}\right)^{2}}+\frac{c_{4213}^{(\mathrm{np})} N_{4213}^{(\mathrm{np})}}{\left(\ell_{5}-\ell_{6}+k_{2}\right)^{2}\left(\ell_{5}-\ell_{6}-k_{4}\right)^{2}}\right)\left.\right|_{\ell_{5}^{*}, \ell_{6}^{*}} .
\end{aligned}
$$

The sum runs over the seven contributing diagrams, following the order displayed in figure 7. The denominators are the two propagators that are left uncut in each diagram when performing this cut. One of the terms in the cut equation, for example, is

$$
\begin{aligned}
\frac{N_{3214}^{(\mathrm{np})}}{\left(\ell_{6}+k_{2}\right)^{2}\left(\ell_{5}-\ell_{6}-k_{4}\right)^{2}}= & \frac{1}{\left(\ell_{6}+k_{2}\right)^{2}\left(\ell_{5}-\ell_{6}-k_{4}\right)^{2}} \\
& \times\left[\left(a_{1,1}^{(\mathrm{np})} \mathrm{PT}(3214)+a_{1,2}^{(\mathrm{np})} \mathrm{PT}(4213)\right) t u\left(\ell_{6}+k_{1}+k_{2}\right)^{2}\right. \\
& \left.+\left(a_{2,1}^{(\mathrm{np})} \mathrm{PT}(3214)+a_{2,2}^{(\mathrm{np})} \mathrm{PT}(4213)\right) s t\left(\ell_{6}+k_{2}+k_{4}\right)^{2}\right] .
\end{aligned}
$$

This has been relabeled from the master labels of eq. (4.4) to the labels of the third nonplanar diagram in figure 7 , including the two uncut propagators. Specifically $\ell_{5} \mapsto-\ell_{6}-$ $k_{2}$ and $\ell_{6} \mapsto-\ell_{5}-k_{1}$ is the relabeling for this diagram. A key simplifying feature is that the $a_{i, j}^{(x)}$ coefficients do not change under this relabeling so as to maintain crossing symmetry; the same four coefficients contribute to all five of the nonplanar double boxes that appear, for example. As discussed in section 3.3.2, the Parke-Taylor factors that appear in eq. (4.8) do not necessarily need to be in the chosen basis, although here $\mathrm{PT}(3214)=\mathrm{PT}_{1}$ and $\mathrm{PT}(4213)=\mathrm{PT}_{2}$.

The single zero condition eq. (4.7) determines five of the six $a_{i j}^{(x)}$ parameters. This is, consistent with our conjecture above, the maximum amount of information that we can extract from all zero conditions. To do so in this example, we reduce to the two-member Parke-Taylor basis mentioned before, and also use Jacobi identities to reduce the seven contributing color factors to a basis of four. Since the remaining Parke-Taylor and color factors are independent, setting the coefficients of $\mathrm{PT} \cdot c$ to zero yields eight potentially 


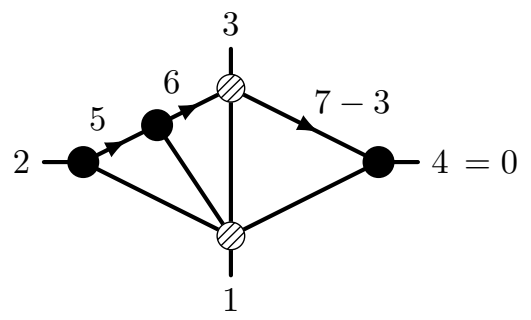

Figure 8. The three-loop four-point MHV amplitude vanishes on this cut. The five-point tree at the bottom of the diagram has $k=2$ or $k=3$, so the overall helicity counting is $k=3$ or $k=4$.

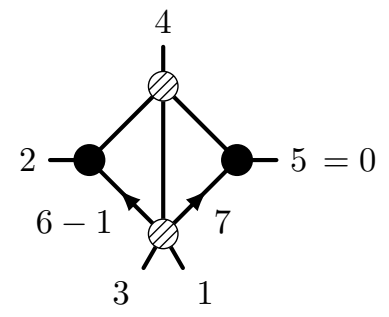

Figure 9. The two-loop five-point MHV amplitude vanishes on this cut. The five-point tree at the bottom of the diagram has $k=2$ or $k=3$, so the overall helicity counting is $k=3$ or $k=4$.

independent equations for the six coefficients. It turns out only five are independent:

$$
\begin{aligned}
& a_{1,2}^{(\mathrm{p})}=a_{1,1}^{(\mathrm{p})}+3 a_{1,1}^{(\mathrm{np})}+a_{2,1}^{(\mathrm{np})}=a_{1,1}^{(\mathrm{p})}+a_{1,1}^{(\mathrm{np})}+a_{2,1}^{(\mathrm{np})}=0, \\
& 2 a_{1,2}^{(\mathrm{p})}-a_{1,1}^{(\mathrm{np})}+a_{1,2}^{(\mathrm{np})}-a_{2,1}^{(\mathrm{np})}+a_{2,2}^{(\mathrm{np})}=a_{1,2}^{(\mathrm{p})}+a_{1,1}^{(\mathrm{np})}+a_{1,2}^{(\mathrm{np})}-a_{2,1}^{(\mathrm{np})}+3 a_{2,2}^{(\mathrm{np})}=0 .
\end{aligned}
$$

The solution for this system is

$$
a_{1,2}^{(\mathrm{p})}=a_{1,1}^{(\mathrm{np})}=a_{2,2}^{(\mathrm{np})}=0, \quad a_{1,2}^{(\mathrm{np})}=a_{2,1}^{(\mathrm{np})}=-a_{1,1}^{(\mathrm{p})},
$$

and any of $a_{1,2}^{(\mathrm{np})}, a_{2,1}^{(\mathrm{np})}$, or $a_{1,1}^{(\mathrm{p})}$ is the overall undetermined parameter. This matches the result in eq. (3.17), if we take $a_{1,1}^{(\mathrm{p})}=1$. This last condition is exactly the overall scale that the zero conditions cannot determine.

Finally, we confirmed that the three-loop four-point and two-loop five-point amplitudes can also be uniquely determined via a zero cut condition up to a single overall constant. We used the cut in figure 8 to determine the arbitrary parameters in the three-loop four-point amplitude, and we used the cut in figure 9 to determine the parameters of the two-loop fivepoint amplitude. We also confirmed in both cases that using one cut where the amplitude does not vanish is sufficient to determine the overall unfixed parameter to the correct value. To confirm that the so-constructed amplitudes are correct, we verified a complete set of unitarity cuts needed to fully determine the amplitudes, matching to the corresponding cuts of previously known results in refs. [51, 58, 59].

We thus conclude that in all three examples that we analyzed, the coefficients in the expansion eq. (3.6) are determined up to one constant by zero conditions. The set of relations is more complicated in the three-loop four-point and two-loop five-point examples than in 
the two-loop four-point example, but in all cases all coefficients are determined as simple rational numbers without any kinematic dependence, leaving one overall coefficient free.

While far from a proof, these results point to the existence of an amplituhedron-like construction in the nonplanar sector of the theory. As discussed in section 2, in the planar sector the existence of such a construction implies that homogeneous conditions determine the amplitudes up to an overall normalization. This is indeed what we have found in the various nonplanar examples studied above: the homogeneous requirements of only logarithmic singularities, no poles at infinity and vanishing of unphysical cuts do determine the amplitudes. In any case, the notion that homogeneous conditions fully determine amplitudes opens a door to applying these ideas to other theories where no geometric properties are expected. Of course, we ultimately would like a direct amplituhedron-like geometric formulation of $\mathcal{N}=4 \mathrm{SYM}$ amplitudes, including the nonplanar contributions. As a next step we would need sensible global variables that allow us to define a unique integrand.

\section{Conclusion}

In this paper we found evidence that an amplituhedron-like construction of nonplanar $\mathcal{N}=4$ SYM theory scattering amplitudes may exist. We did so by checking the expected consequences of such a construction: that the integrand should be determined by homogeneous conditions, such as vanishing on certain cut solutions. We also gave additional nontrivial evidence for the conjecture that only logarithmic singularities appear in nonplanar amplitudes [52, 53], which is another characteristic feature of planar amplitudes resulting from the amplituhedron construction.

An important complication is that unlike in the planar sector, there is no unique integrand of scattering amplitudes which can be directly interpreted as a volume of some space. This forced us to chop up the amplitude into local diagrams containing only Feynman propagators. As pointed out in ref. [52] and further developed in ref. [53], analytic properties that follow from dual conformal invariance can be imposed on such local diagrams. We developed the notion of a pure integrand basis: a basis of integrands with only logarithmic singularities, no poles at infinity and only unit leading singularities. The first property is motivated by the analogous statement for on-shell diagrams in $\mathcal{N}=4 \mathrm{SYM}$. If, like in the planar case, we understood how to formulate nonplanar recursion relations, we expect that it would then be possible to express nonplanar amplitudes directly as sums of on-shell diagrams $[25,56,78]$ and manifestly expose their $d \log$ structure. The latter two properties lift the exact content of dual conformal symmetry in the planar sector to the nonplanar one.

We constructed a pure integrand basis for each of the two-loop four-point, three-loop four-point and two-loop five-point amplitudes, and showed that the amplitudes could be expanded in their respective bases. This confirmed that the three example amplitudes share the three properties of the pure integrands. Our pure integrand representations here are closely related to refs. $[52,53]$ for four-point amplitudes at two- and three-loops, while our representation of the two-loop five-point amplitude has completely novel properties compared to the result in ref. [59]. The fact that we exposed analytic properties in the 
nonplanar sector similar to those connected to dual conformal symmetry in the planar sector suggests that an analog of dual conformal symmetry may exist in the nonplanar sector. (For Yangian symmetry a similar statement is less clear.)

One particularly bold future goal is to lift the amplituhedron [36] paradigm from the planar sector to the nonplanar sector of $\mathcal{N}=4$ SYM. The amplituhedron provides a geometric picture of the planar integrand where all standard physical principles like locality and unitarity are derived. In such a picture, traditional ways of organizing amplitudes, be it via Feynman diagrams, unitarity cuts, or even on-shell diagrams, are consequences of amplituhedron geometry, rather than a priori organizational principles. The amplituhedron reverses traditional logic, as logarithmic singularities and dual conformal symmetry, rather than locality and unitarity, are fundamental inputs into the definition of the amplituhedron. The definition then invokes intuitive geometric ideas about the inside of a projective triangle, generalized to the more complicated setting of Grassmannian geometry.

We would like to carry this geometric picture over to the nonplanar sector. However, a lack of global variables limits us to demanding that the amplitude be a sum of local integrals. This already imposes locality and some unitarity constraints. Nevertheless, after imposing special analytic structures on the basis integrals - unit logarithmic singularities with no poles at infinity - one can extract the "remaining" geometric information. Motivated by the discussion in ref. [40], in this paper we conjectured that this remaining information is a set of zero conditions, i.e. cuts on which the amplitude vanishes. This is exactly the statement which we successfully carried over to the nonplanar sector and tested in examples in section 4 . Here we propose that after constructing a pure integrand basis, zero conditions are sufficient for finding the complete amplitude.

This provides nontrivial evidence that an amplituhedron-like construction might very well exist beyond the planar limit for amplitudes in $\mathcal{N}=4 \mathrm{SYM}$ theory. However there are still many obstacles including, among other things, a choice of good variables and a geometric space in which nonplanar scattering amplitudes are defined as volumes. If such a nonplanar amplituhedron really exists, it would be phrased in terms of very interesting mathematical structures going beyond those of the planar amplituhedron.

If our zero condition conjecture indeed holds, how might it extend to other theories? The most naive possibility is that $\mathcal{N}=4 \mathrm{SYM}$ amplitudes are the most constrained amplitudes and so need no inhomogeneous conditions except for overall normalization, while amplitudes in other theories, with less supersymmetry for example, do need additional inhomogeneous information. Even in such theories the zero conditions would still constrain the amplitudes, and it would be interesting to see which and how many additional inhomogeneous conditions are required to completely determine the amplitudes.

It may be possible to link the $\mathcal{N}=4 \mathrm{SYM}$ results we presented here directly to identical helicity amplitudes in quantum chromodynamics (QCD) via dimension shifting relations $[79,80]$. These relations were recently employed to aid in the construction of a representation of the two-loop five-point identical helicity QCD amplitude where the duality between color and kinematics holds [81]. It should also be possible to find a new representation of the identical helicity QCD amplitude in terms of the $\mathcal{N}=4 \mathrm{SYM}$ representation we gave here. 
Another line of research is to concentrate on individual integrals rather than on the full amplitude. After integration, integrands with only logarithmic singularities are expected to have uniform maximum transcendental weight at the loop order of the integrand [82]. This provides a nice connection between properties of the integrand and conjectured properties of final integrated amplitudes. On the practical level, having a good basis of master integrals under integral reduction is important for many problems, including applications to phenomenology. As explained in refs. [83, 84], uniformly transcendental integrals obey relatively simple differential equations, making them easy to work with $[85,86]$. This also makes our basis of pure integrands useful for five-point scattering in NNLO QCD. For a recent discussion of the planar case see ref. [87].

As already noted in ref. [53], the types of gauge-theory results described here can have a direct bearing on issues in quantum gravity, through the double-copy relation of YangMills theories to gravity [51]. We expect that developing a better understanding of the nonplanar sector of $\mathcal{N}=4 \mathrm{SYM}$ will aid our ability to construct corresponding gravity amplitudes, where no natural separation of planar and nonplanar contributions exists.

In summary, we have presented evidence that nonplanar integrands of $\mathcal{N}=4 \mathrm{SYM}$ share important analytic structure with planar ones. We have also presented evidence for a geometric structure similar to the amplituhedron [36] based on the idea that such a structure implies that zero conditions are sufficient to fix the amplitude, up to an overall normalization. While there is much more to do, these results suggest that the full theory has structure at least as rich as the planar theory.

\section{Acknowledgments}

This work was supported in part by the US Department of Energy under Award Number DE-SC0009937 and DE-SC0011632. J. T. is supported in part by the David and Ellen Lee Postdoctoral Scholarship. E. H. is supported in part by a Dominic Orr Graduate Fellowship. Z. B. is grateful to the Simons Foundation for support.

Open Access. This article is distributed under the terms of the Creative Commons Attribution License (CC-BY 4.0), which permits any use, distribution and reproduction in any medium, provided the original author(s) and source are credited.

\section{References}

[1] J. Drummond, J. Henn, V. Smirnov and E. Sokatchev, Magic identities for conformal four-point integrals, JHEP 01 (2007) 064 [hep-th/0607160].

[2] L.F. Alday and J.M. Maldacena, Gluon scattering amplitudes at strong coupling, JHEP 06 (2007) 064 [arXiv:0705.0303] [INSPIRE].

[3] J.M. Drummond, J. Henn, G.P. Korchemsky and E. Sokatchev, Dual superconformal symmetry of scattering amplitudes in $N=4$ super-Yang-Mills theory, Nucl. Phys. B 828 (2010) 317 [arXiv:0807.1095] [INSPIRE].

[4] J.M. Drummond, J.M. Henn and J. Plefka, Yangian symmetry of scattering amplitudes in $N=4$ super Yang-Mills theory, JHEP 05 (2009) 046 [arXiv:0902.2987] [INSPIRE]. 
[5] N. Beisert and M. Staudacher, The N=4 SYM integrable super spin chain, Nucl. Phys. B 670 (2003) 439 [hep-th/0307042] [INSPIRE].

[6] N. Beisert, B. Eden and M. Staudacher, Transcendentality and crossing, J. Stat. Mech. 0701 (2007) P01021 [hep-th/0610251] [INSPIRE].

[7] J.M. Drummond, G.P. Korchemsky and E. Sokatchev, Conformal properties of four-gluon planar amplitudes and Wilson loops, Nucl. Phys. B 795 (2008) 385 [arXiv:0707.0243] [INSPIRE].

[8] A. Brandhuber, P. Heslop and G. Travaglini, $M H V$ amplitudes in $N=4$ super Yang-Mills and Wilson loops, Nucl. Phys. B 794 (2008) 231 [arXiv:0707.1153] [INSPIRE].

[9] J.M. Drummond, J. Henn, G.P. Korchemsky and E. Sokatchev, Conformal Ward identities for Wilson loops and a test of the duality with gluon amplitudes, Nucl. Phys. B 826 (2010) 337 [arXiv:0712.1223] [INSPIRE].

[10] L.J. Mason and D. Skinner, The complete planar S-matrix of $N=4 S Y M$ as a Wilson loop in twistor space, JHEP 12 (2010) 018 [arXiv: 1009.2225] [INSPIRE].

[11] S. Caron-Huot, Notes on the scattering amplitude/Wilson loop duality, JHEP 07 (2011) 058 [arXiv: 1010.1167] [INSPIRE].

[12] L.F. Alday, B. Eden, G.P. Korchemsky, J. Maldacena and E. Sokatchev, From correlation functions to Wilson loops, JHEP 09 (2011) 123 [arXiv:1007.3243] [INSPIRE].

[13] B. Basso, A. Sever and P. Vieira, Spacetime and flux tube S-matrices at finite coupling for $N=4$ supersymmetric Yang-Mills theory, Phys. Rev. Lett. 111 (2013) 091602 [arXiv:1303.1396] [INSPIRE].

[14] B. Basso, J. Caetano, L. Cordova, A. Sever and P. Vieira, OPE for all helicity amplitudes, JHEP 08 (2015) 018 [arXiv: 1412.1132] [INSPIRE].

[15] B. Basso, A. Sever and P. Vieira, Hexagonal Wilson loops in planar $\mathcal{N}=4$ SYM theory at finite coupling, arXiv:1508.0304.

[16] L.J. Dixon, J.M. Drummond and J.M. Henn, Bootstrapping the three-loop hexagon, JHEP 11 (2011) 023 [arXiv: 1108.4461] [INSPIRE].

[17] L.J. Dixon, J.M. Drummond and J.M. Henn, Analytic result for the two-loop six-point NMHV amplitude in $N=4$ super Yang-Mills theory, JHEP 01 (2012) 024 [arXiv:1111.1704] [INSPIRE].

[18] L.J. Dixon, J.M. Drummond, M. von Hippel and J. Pennington, Hexagon functions and the three-loop remainder function, JHEP 12 (2013) 049 [arXiv: 1308.2276] [INSPIRE].

[19] L.J. Dixon and M. von Hippel, Bootstrapping an NMHV amplitude through three loops, JHEP 10 (2014) 065 [arXiv: 1408.1505] [INSPIRE].

[20] L.J. Dixon, M. von Hippel and A.J. McLeod, The four-loop six-gluon NMHV ratio function, arXiv: 1509.0812.

[21] A.B. Goncharov, M. Spradlin, C. Vergu and A. Volovich, Classical polylogarithms for amplitudes and Wilson loops, Phys. Rev. Lett. 105 (2010) 151605 [arXiv:1006.5703] [INSPIRE].

[22] J. Golden, A.B. Goncharov, M. Spradlin, C. Vergu and A. Volovich, Motivic amplitudes and cluster coordinates, JHEP 01 (2014) 091 [arXiv: 1305.1617] [INSPIRE]. 
[23] J.M. Drummond, G. Papathanasiou and M. Spradlin, A symbol of uniqueness: the cluster bootstrap for the 3-loop MHV heptagon, JHEP 03 (2015) 072 [arXiv:1412.3763] [INSPIRE].

[24] D. Parker, A. Scherlis, M. Spradlin and A. Volovich, Hedgehog bases for $A_{n}$ cluster polylogarithms and an application to six-point amplitudes, JHEP 11 (2015) 136 [arXiv: 1507.0195].

[25] N. Arkani-Hamedet al., Scattering amplitudes and the positive grassmannian, arXiv: 1212.5605.

[26] N. Arkani-Hamed, F. Cachazo, C. Cheung and J. Kaplan, A duality for the S matrix, JHEP 03 (2010) 020 [arXiv: 0907.5418] [inSPIRE].

[27] N. Arkani-Hamed, F. Cachazo and C. Cheung, The grassmannian origin of dual superconformal invariance, JHEP 03 (2010) 036 [arXiv:0909.0483] [INSPIRE].

[28] L.J. Mason and D. Skinner, Dual superconformal invariance, momentum twistors and grassmannians, JHEP 11 (2009) 045 [arXiv:0909.0250] [INSPIRE].

[29] N. Arkani-Hamed, J. Bourjaily, F. Cachazo and J. Trnka, Unification of residues and grassmannian dualities, JHEP 01 (2011) 049 [arXiv:0912.4912] [INSPIRE].

[30] N. Arkani-Hamed, J. Bourjaily, F. Cachazo and J. Trnka, Local spacetime physics from the grassmannian, JHEP 01 (2011) 108 [arXiv:0912.3249] [INSPIRE].

[31] N. Arkani-Hamed, J.L. Bourjaily, F. Cachazo, S. Caron-Huot and J. Trnka, The all-loop integrand for scattering amplitudes in planar $N=4$ SYM, JHEP 01 (2011) 041 [arXiv: 1008.2958] [INSPIRE].

[32] Y.-T. Huang and C. Wen, ABJM amplitudes and the positive orthogonal grassmannian, JHEP 02 (2014) 104 [arXiv: 1309.3252] [INSPIRE].

[33] Y.-t. Huang, C. Wen and D. Xie, The positive orthogonal grassmannian and loop amplitudes of ABJM, J. Phys. A 47 (2014) 474008 [arXiv:1402.1479] [InSPIRE].

[34] J. Kim and S. Lee, Positroid stratification of orthogonal grassmannian and ABJM amplitudes, JHEP 09 (2014) 085 [arXiv: 1402.1119] [INSPIRE].

[35] H. Elvang et al., Grassmannians for scattering amplitudes in $4 D \mathcal{N}=4 S Y M$ and $3 D$ ABJM, JHEP 12 (2014) 181 [arXiv:1410.0621] [INSPIRE].

[36] N. Arkani-Hamed and J. Trnka, The amplituhedron, JHEP 10 (2014) 030 [arXiv: 1312.2007] [INSPIRE].

[37] N. Arkani-Hamed and J. Trnka, Into the amplituhedron, JHEP 12 (2014) 182 [arXiv: 1312.7878] [INSPIRE].

[38] S. Franco, D. Galloni, A. Mariotti and J. Trnka, Anatomy of the amplituhedron, JHEP 03 (2015) 128 [arXiv:1408.3410] [INSPIRE].

[39] Y. Bai and S. He, The amplituhedron from momentum twistor diagrams, JHEP 02 (2015) 065 [arXiv: 1408.2459] [INSPIRE].

[40] N. Arkani-Hamed, A. Hodges and J. Trnka, Positive amplitudes in the amplituhedron, JHEP 08 (2015) 030 [arXiv: 1412.8478] [INSPIRE].

[41] T. Lam, Amplituhedron cells and Stanley symmetric functions, Commun. Math. Phys. 343 (2016) 1025 [arXiv: 1408.5531] [INSPIRE]. 
[42] Y. Bai, S. He and T. Lam, The amplituhedron and the one-loop Grassmannian measure, arXiv: 1510.0355.

[43] L. Ferro, T. Lukowski, A. Orta and M. Parisi, Towards the amplituhedron volume, arXiv: 1512.0495.

[44] G. Lusztig, Total positivity in partial flag manifolds, Represent. Theory 2 (1998) 70.

[45] A. Postnikov, Total positivity, Grassmannians and networks, math/0609764 [INSPIRE].

[46] A. Postnikov, D. Speyer and L. Williams, Matching polytopes, toric geometry, and the non-negative part of the Grassmannian, arXiv:0706.2501.

[47] L.K. Williams, Enumeration of totally positive Grassmann cells, math/0307271.

[48] A.B. Goncharov and R. Kenyon, Dimers and cluster integrable systems, arXiv:1107.5588 [INSPIRE].

[49] A. Knutson, T. Lam and D. Speyer, Positroid varieties: juggling and geometry, arXiv:1111.3660.

[50] Z. Bern, J.J.M. Carrasco and H. Johansson, New relations for gauge-theory amplitudes, Phys. Rev. D 78 (2008) 085011 [arXiv:0805.3993] [INSPIRE].

[51] Z. Bern, J.J.M. Carrasco and H. Johansson, Perturbative quantum gravity as a double copy of gauge theory, Phys. Rev. Lett. 105 (2010) 061602 [arXiv: 1004.0476] [INSPIRE].

[52] N. Arkani-Hamed, J.L. Bourjaily, F. Cachazo and J. Trnka, Singularity structure of maximally supersymmetric scattering amplitudes, Phys. Rev. Lett. 113 (2014) 261603 [arXiv: 1410.0354] [INSPIRE].

[53] Z. Bern, E. Herrmann, S. Litsey, J. Stankowicz and J. Trnka, Logarithmic singularities and maximally supersymmetric amplitudes, JHEP 06 (2015) 202 [arXiv: 1412.8584] [INSPIRE].

[54] S.J. Parke and T.R. Taylor, An amplitude for $n$ gluon scattering, Phys. Rev. Lett. 56 (1986) 2459 [INSPIRE].

[55] M.L. Mangano, S.J. Parke and Z. Xu, Duality and multi-gluon scattering, Nucl. Phys. B 298 (1988) 653 [INSPIRE].

[56] N. Arkani-Hamed, J.L. Bourjaily, F. Cachazo, A. Postnikov and J. Trnka, On-shell structures of MHV amplitudes beyond the planar limit, JHEP 06 (2015) 179 [arXiv:1412.8475] [INSPIRE].

[57] L.J. Dixon, A.J. McLeod, J. Trnka and M. von Hippel, to appear.

[58] Z. Bern et al., Three-Loop Superfiniteness of $N=8$ Supergravity, Phys. Rev. Lett. 98 (2007) 161303 [hep-th/0702112] [INSPIRE].

[59] J.J. Carrasco and H. Johansson, Five-point amplitudes in $N=4$ super-Yang-Mills theory and $N=8$ supergravity, Phys. Rev. D 85 (2012) 025006 [arXiv:1106.4711] [INSPIRE].

[60] Z. Bern, J.J. Carrasco, T. Dennen, Y.-t. Huang and H. Ita, Generalized unitarity and six-dimensional helicity, Phys. Rev. D 83 (2011) 085022 [arXiv: 1010.0494] [INSPIRE].

[61] Z. Bern et al., The two-loop six-gluon MHV amplitude in maximally supersymmetric Yang-Mills theory, Phys. Rev. D 78 (2008) 045007 [arXiv: 0803.1465] [INSPIRE].

[62] Z. Bern, L.J. Dixon, D.C. Dunbar and D.A. Kosower, One loop n point gauge theory amplitudes, unitarity and collinear limits, Nucl. Phys. B 425 (1994) 217 [hep-ph/9403226] [INSPIRE]. 
[63] Z. Bern, L.J. Dixon, D.C. Dunbar and D.A. Kosower, Fusing gauge theory tree amplitudes into loop amplitudes, Nucl. Phys. B 435 (1995) 59 [hep-ph/9409265] [INSPIRE].

[64] Z. Bern, J.J.M. Carrasco, H. Johansson and D.A. Kosower, Maximally supersymmetric planar Yang-Mills amplitudes at five loops, Phys. Rev. D 76 (2007) 125020 [arXiv:0705.1864] [INSPIRE].

[65] R. Britto, F. Cachazo, B. Feng and E. Witten, Direct proof of tree-level recursion relation in Yang-Mills theory, Phys. Rev. Lett. 94 (2005) 181602 [hep-th/0501052] [INSPIRE].

[66] A. Hodges, Eliminating spurious poles from gauge-theoretic amplitudes, JHEP 05 (2013) 135 [arXiv: 0905.1473] [INSPIRE].

[67] F. Cachazo, Sharpening the leading singularity, arXiv:0803.1988 [INSPIRE].

[68] J.M. Drummond, J. Henn, G.P. Korchemsky and E. Sokatchev, The hexagon Wilson loop and the BDS ansatz for the six-gluon amplitude, Phys. Lett. B 662 (2008) 456 [arXiv:0712.4138] [INSPIRE].

[69] N. Arkani-Hamed, J.L. Bourjaily, F. Cachazo and J. Trnka, Local integrals for planar scattering amplitudes, JHEP 06 (2012) 125 [arXiv:1012.6032] [INSPIRE].

[70] E. Witten, Perturbative gauge theory as a string theory in twistor space, Commun. Math. Phys. 252 (2004) 189 [hep-th/0312171] [INSPIRE].

[71] J.L. Bourjaily, S. Caron-Huot and J. Trnka, Dual-conformal regularization of infrared loop divergences and the chiral box expansion, JHEP 01 (2015) 001 [arXiv:1303.4734] [INSPIRE].

[72] J.L. Bourjaily and J. Trnka, Local integrand representations of all two-loop amplitudes in planar SYM, JHEP 08 (2015) 119 [arXiv:1505.0588].

[73] Z. Bern, J.S. Rozowsky and B. Yan, Two loop four gluon amplitudes in $N=4$ super Yang-Mills, Phys. Lett. B 401 (1997) 273 [hep-ph/9702424] [INSPIRE].

[74] R. Kleiss and H. Kuijf, Multi-gluon cross-sections and five jet production at hadron colliders, Nucl. Phys. B 312 (1989) 616 [INSPIRE].

[75] Z. Bern, L.J. Dixon, D.C. Dunbar, M. Perelstein and J.S. Rozowsky, On the relationship between Yang-Mills theory and gravity and its implication for ultraviolet divergences, Nucl. Phys. B 530 (1998) 401 [hep-th/9802162] [INSPIRE].

[76] Z. Bern, J.J.M. Carrasco, L.J. Dixon, H. Johansson and R. Roiban, Simplifying multiloop integrands and ultraviolet divergences of gauge theory and gravity amplitudes, Phys. Rev. D 85 (2012) 105014 [arXiv:1201.5366] [INSPIRE].

[77] M.L. Mangano and S.J. Parke, Multiparton amplitudes in gauge theories, Phys. Rept. 200 (1991) 301 [hep-th/0509223] [INSPIRE].

[78] S. Franco, D. Galloni, B. Penante, and C. Wen, Non-planar on-shell diagrams, JHEP 06 (2015) 199 [arXiv: 1502.0203].

[79] Z. Bern, L.J. Dixon, D.C. Dunbar and D.A. Kosower, One loop selfdual and $N=4$ super Yang-Mills, Phys. Lett. B 394 (1997) 105 [hep-th/9611127] [INSPIRE].

[80] Z. Bern, L.J. Dixon, M. Perelstein and J.S. Rozowsky, Multileg one loop gravity amplitudes from gauge theory, Nucl. Phys. B 546 (1999) 423 [hep-th/9811140] [INSPIRE].

[81] G. Mogull and D. O'Connell, Overcoming obstacles to colour-kinematics duality at two loops, arXiv: 1511.0665. 
[82] A.V. Kotikov, L.N. Lipatov, A.I. Onishchenko and V.N. Velizhanin, Three loop universal anomalous dimension of the Wilson operators in $N=4$ SUSY Yang-Mills model, Phys. Lett. B 595 (2004) 521 [Erratum ibid. B 632 (2006) 754] [hep-th/0404092] [INSPIRE].

[83] J.M. Henn, Multiloop integrals in dimensional regularization made simple, Phys. Rev. Lett. 110 (2013) 251601 [arXiv: 1304.1806] [INSPIRE].

[84] J.M. Henn, A.V. Smirnov and V.A. Smirnov, Analytic results for planar three-loop four-point integrals from a Knizhnik-Zamolodchikov equation, JHEP 07 (2013) 128 [arXiv:1306.2799] [INSPIRE].

[85] S. Caron-Huot and J.M. Henn, Iterative structure of finite loop integrals, JHEP 06 (2014) 114 [arXiv: 1404.2922] [INSPIRE].

[86] J.M. Henn, Lectures on differential equations for Feynman integrals, J. Phys. A 48 (2015) 153001 [arXiv: 1412.2296] [INSPIRE].

[87] T. Gehrmann, J.M. Henn and N.A. Lo Presti, Analytic form of the two-loop planar five-gluon all-plus-helicity amplitude in QCD, arXiv:1511.0540. 